\title{
Article
}

\section{The Wolf-Rayet population of the nearby barred spiral galaxy NGC 5068 uncovered by the Very Large Telescope and Gemini}

Bibby, Joanne and Crowther, P. A.

Available at http://clok.uclan.ac.uk/7295/

Bibby, Joanne and Crowther, P. A. (2012) The Wolf-Rayet population of the nearby barred spiral galaxy NGC 5068 uncovered by the Very Large Telescope and Gemini. Monthly Notices of the Royal Astronomical Society, 420 (4). pp. 3091-3107. ISSN 00358711

It is advisable to refer to the publisher's version if you intend to cite from the work. http://dx.doi.org/10.1111/j.1365-2966.2011.20220.x

For more information about UCLan's research in this area go to http://www.uclan.ac.uk/researchgroups/ and search for <name of research Group>.

For information about Research generally at UCLan please go to http://www.uclan.ac.uk/research/

All outputs in CLoK are protected by Intellectual Property Rights law, including Copyright law. Copyright, IPR and Moral Rights for the works on this site are retained by the individual authors and/or other copyright owners. Terms and conditions for use of this material are defined in the policies page. 


\title{
The Wolf-Rayet population of the nearby barred spiral galaxy NGC 5068 uncovered by the Very Large Telescope and Gemini
}

\author{
J. L. Bibby ${ }^{1,2 \star}$ and P. A. Crowther ${ }^{2}$ \\ ${ }^{1}$ Department of Astrophysics, American Museum of Natural History, 79th Street and Central Park West, New York, NY 10024-5192, USA \\ ${ }^{2}$ Department of Physics and Astronomy, University of Sheffield, Hicks Building, Hounsfield Road, Sheffield S3 7RH
}

Accepted 2011 November 16. Received 2011 November 16; in original form 2011 September 29

\begin{abstract}
We present a narrow-band Very Large Telescope/Focal Reduced Low-dispersion Spectrograph \#1 imaging survey of the SAB(rs)cd spiral galaxy NGC 5068, located at a distance of $5.45 \mathrm{Mpc}$, from which 160 candidate Wolf-Rayet sources have been identified, of which 59 cases possess statistically significant $\lambda 4686$ excesses. Follow-up Gemini Multi-Object Spectrograph spectroscopy of 64 candidates, representing 40 per cent of the complete photometric catalogue, confirms Wolf-Rayet signatures in 30 instances, corresponding to a 47 per cent success rate. 21 out of 22 statistically significant photometric sources are spectroscopically confirmed. Nebular emission detected in 30 per cent of the Wolf-Rayet candidates spectrally observed, which enable a re-assessment of the metallicity gradient in NGC 5068. A central metallicity of $\log (\mathrm{O} / \mathrm{H})+12 \sim 8.74$ is obtained, declining to 8.23 at $R_{25}$. We combine our spectroscopy with archival $\mathrm{H} \alpha$ images of NGC 5068 to estimate a current star formation rate of $0.63_{-0.13}^{+0.11} \mathrm{M}_{\odot} \mathrm{yr}^{-1}$, and provide a catalogue of the 28 brightest $\mathrm{H}$ II regions from our own continuum subtracted $\mathrm{H} \alpha$ images, of which $\sim 17$ qualify as giant $\mathrm{H}$ II regions. Spectroscopically, we identify $24 \mathrm{WC}$ - and $18 \mathrm{WN}$-type Wolf-Rayet stars within 30 sources since emission-line fluxes indicate multiple Wolf-Rayet stars in several cases. We estimate an additional $\sim 66$ Wolf-Rayet stars from the remaining photometric candidates, although sensitivity limits will lead to an incomplete census of visually faint WN stars, from which we estimate a global population of $\sim 170$ Wolf-Rayet stars. Based on the $\mathrm{H} \alpha$-derived O star population of NGC 5068 and $N(\mathrm{WR}) / N(\mathrm{O}) \sim 0.03$, representative of the Large Magellanic Cloud, we would expect a larger Wolf-Rayet population of 270 stars. Finally, we have compared the spatial distribution of spectroscopically confirmed WN and WC stars with Sloan Digital Sky Survey derived supernovae, and find both WN and WC stars to be most consistent with the parent population of Type Ib supernovae.
\end{abstract}

Key words: supernovae: general - stars: Wolf-Rayet - H II regions - galaxies: individual: NGC 5068 - galaxies: stellar content.

\section{INTRODUCTION}

Massive stars $\left(>8 \mathrm{M}_{\odot}\right)$ dominate the radiative ionizing budget of star-forming galaxies, and contribute significantly to the mechanical energy budget via their powerful stellar winds and ultimate death as core-collapse supernovae (ccSNe), plus the chemical enrichment of galaxies, especially for $\alpha$ elements. However, theoretically their evolutionary paths and precise fate remain unclear, arising from details of stellar winds, initial rotation and metallicity. Empirical results such as the ratio of blue supergiant (BSG) and red supergiant (RSG), or the ratio of Wolf-Rayet (WR) to O stars provide sensi-

^E-mail: jbibby@amnh.org tive tests of evolutionary models which incorporate complex processes, such as rotation (Langer \& Maeder 1995; Maeder \& Meynet 2000).

The conventional WR phase corresponds to the core-helium burning stage of massive stars $\left(\geq 20-25 \mathrm{M}_{\odot}\right.$ in the Milky Way), with a duration of only a few hundred thousand years (Crowther 2007). WR stars possess winds densities which are an order of magnitude higher than $\mathrm{O}$ stars, producing a unique, broad emission-line spectrum. Spectroscopically, they are divided into WN and WC subtypes which are dominated by $\mathrm{He}_{\text {II }} \lambda 4686$ and $\mathrm{C}_{\text {III }} \lambda 4650+\mathrm{C}_{\text {IV }}$ $\lambda 5808$ emission lines, respectively. Consequently, WN and WC stars are associated with the products of the core hydrogen- (CNO cycle) and helium burning ( $3 \alpha)$. Photometrically, WR stars cannot be distinguished from BSG via broad-band imaging, although strong 
emission lines facilitate their detection via suitable narrow-band filters (Moffat \& Shara 1983).

Follow-up spectroscopy of photometric candidates with 4-m aperture telescopes is routinely capable of determining the nature of WR stars in Local Group galaxies (Neugent \& Massey 2011). However, beyond the Local Group 8-m class telescopes are required. The advantages of using telescopes such as the Very Large Telescope (VLT) were first demonstrated by Schild et al. (2003) in their investigation into the WR population of NGC 300. Observations of massive stars beyond $\sim 1 \mathrm{Mpc}$ provide a broader range of galaxy morphological types and metallicities than those available in the Local Group. In addition, the much larger volume sampled enables progress in empirically linking various flavours of ccSNe to progenitor stars.

Massive stars with initial masses $\sim 8-20 \mathrm{M}_{\odot}$ retain their hydrogen envelope and end their lives in the RSG phase undergoing core-collapse and producing a H-rich Type II ccSN (Smartt 2009). This has been observationally confirmed from archival broad-band pre-SN images, e.g. SN 2003gd, which was identified as a $\sim 8 \mathrm{M}_{\odot}$ RSG (Smartt et al. 2004). Stars with initial masses above $\sim 20 \mathrm{M}_{\odot}$ are thought to end their lives during the WR phase as $\mathrm{H}$-poor Type $\mathrm{Ib}$ or H + He-poor Type Ic ccSNe (Woosley \& Bloom 2006). However, no direct detection of Type $\mathrm{Ib} / \mathrm{c} \mathrm{SNe}$ progenitors have, to date, been established.

A greater understanding of the progenitors of ccSNe can be achieved through observations of the environments in which the SNe occur (Anderson \& James 2008). Kelly, Kirshner \& Pahre (2008) found that H-rich Type II ccSNe follow the distribution of the host galaxy light, whereas Type Ib and Type Ic ccSNe are located in the brightest regions of the galaxy. Moreover, the distributions of Type Ib and Type Ic ccSNe are different, suggesting that they have different progenitors. If WN and WC stars are the progenitors of Type $\mathrm{Ib}$ and Type Ic ccSNe, respectively, then they should be located in the same regions of the galaxy. Leloudas et al. (2010) applied the same method to the spectroscopic WR surveys of M83 and NGC 1313 (Hadfield et al. 2005; Hadfield \& Crowther 2007), confirming that the WN-Type Ib and WC-Type Ic were the most likely progenitor scenarios, albeit with low number statistics.

An alternative scenario for the production of Type Ib/c ccSNe has been proposed by Podsialowski, Joss \& Hsu (1992) in which intermediate-mass stars in binary systems lose their hydrogen and helium envelopes via Roche lobe overflow and/or common envelope evolution. This stripping of outer layers would result in a low-mass helium core which would have WR-like emission, however, such low-mass helium cores would be completely hidden by their higher mass companions. This scenario appears consistent with Type Ic SN 2002ap in M74, for which deep Canada-France-Hawaii Telescope (CFHT) broad-band images did not reveal any progenitor down to a limiting magnitude of $M_{B}=-4.2 \mathrm{mag}$ (Crockett et al. 2007), suggesting a binary system with a low-mass $\mathrm{C}+\mathrm{O}$ core of $\sim 5 \mathrm{M}_{\odot}$ (Mazzali et al. 2002).

At present, the relative statistics of ccSNe (Smartt et al. 2009; Li et al. 2011) favour both binary and single star evolutionary channels (Smith et al. 2011). Our group are undertaking photometric and spectroscopic surveys of WR stars in a dozen star-forming galaxies beyond the Local Group for the purpose of studying massive stars in a variety of environments and to provide a data base of future Type Ib/c progenitors.

In this paper we investigate the WR population of NGC 5068, a face-on barred spiral galaxy $(\mathrm{SAB}(\mathrm{rs}) \mathrm{cd}$; de Vaucouleurs et al. 1991), situated at a distance of 5.45 Mpc (Herrmann et al. 2008) beyond the Centaurus A group (Karachentsev et al. 2007). NGC 5068 is known to host WR stars, since their spectroscopic signatures have been serendipitously detected in observations of bright $\mathrm{H}$ II regions by Rosa \& D'Odorico (1986). However, no systematic search for WR stars in NGC 5068 has been undertaken and the stellar content of NGC 5068 remains largely unknown. Here, we present a comprehensive study of the massive stellar population of NGC 5068, and compare the distribution of WR stars relative to the giant $\mathrm{H}$ II regions in NGC 5068. We constrain estimates of the star formation rate (SFR) of NGC 5068 which vary wildly, from $\sim 0.35$ to $\sim 3 \mathrm{M}_{\odot} \mathrm{yr}^{-1}$ (Ryder \& Dopita 1994; Martin \& Friedli 1997). Similarly, measurements of the central metallicity of the galaxy range from Large Magellanic Cloud (LMC)-like metallicities (Hodge 1974) to supersolar (Ryder 1995).

This paper is organized as follows. In Section 2 we discuss the observations, data reduction and source selection, while in Section 3 we analyse the nebular properties of $\mathrm{H}$ II regions in NGC 5068. Section 4 determines the WR population and Section 5 the $\mathrm{O}$ star population and SFR of NGC 5068. In Section 6 we assess the global WR population based on the completeness of our observations. Section 7 provides a comparison of results with both NGC 7793 and predictions from evolutionary theory. Finally, Section 8 compares the spatial distribution of the WR stars in NGC 5068 with those of ccSN; a summary follows in Section 9.

\section{OBSERVATIONS AND DATA REDUCTION}

NGC 5068 has been imaged with the ESO VLT and Focal Reduced Low-dispersion Spectrograph \#1 (FORS1) covering a field of view of $6.8 \times 6.8 \mathrm{arcmin}^{2}$ with a plate scale of $0.25 \mathrm{arcsec} \mathrm{pixel}^{-1}$. Both broad- and narrow-band imaging were obtained on 2008 April 7 under program ID 081.B-0289 (P.I. Crowther). In addition, the Gemini Multi-Object Spectrograph (GMOS) on the Gemini-South telescope was used to obtain follow-up spectroscopy in 2009 March-April under program ID GS-2009A-Q-20 (P.I. Crowther). The R150 grating was placed at a central wavelength of 510 and $530 \mathrm{~nm}$ with a dispersion of $\sim 3.5 \AA$ pixel $^{-1}$. Further details of the observations can be found in Table 1 .

\subsection{Imaging and photometry}

FORS1 was used on 2008 April 7 to obtain narrow-band imaging of NGC 5068 centred on $\lambda 4684$ and $\lambda 4781$ under good seeing conditions of $\sim 0.8$ arcsec. Three $1025 \mathrm{~s}$ exposures were obtained

Table 1. Observational log of VLT/FORS1 imaging and Gemini/GMOS spectroscopy of NGC 5068. Imaging was taken under program ID 081.B0289 and spectroscopy under program GS-2009A-Q-20.

\begin{tabular}{lcccc}
\hline Date & $\begin{array}{c}\text { Filter/ } \\
\text { mask ID }\end{array}$ & $\begin{array}{c}\lambda_{\mathrm{c}} \\
(\AA)\end{array}$ & $\begin{array}{c}\text { Exposure } \\
\text { time }(\mathrm{s})\end{array}$ & $\begin{array}{c}\text { Seeing } \\
(\operatorname{arcsec})\end{array}$ \\
\hline \multicolumn{5}{c}{ VLT/FORS1 imaging } \\
2008 April 7 & B high & 4400 & 250 & 0.80 \\
& V high & 5570 & 250 & 0.80 \\
& $\mathrm{H} \alpha$ & 6563 & 250 & 0.85 \\
& H $\alpha / 4500$ & 6665 & 250 & 0.75 \\
He II & 4684 & $3 \times 1025$ & 0.80 \\
2009 April 3 & He II/6500 & 4781 & $3 \times 1025$ & 0.75 \\
2009 March 31 & MASK 1 & $5100 / 5300$ & $6 \times 430$ & 0.87 \\
2009 April 1 & MASK 2 & $5100 / 5300$ & $6 \times 1400$ & 0.87 \\
\hline
\end{tabular}




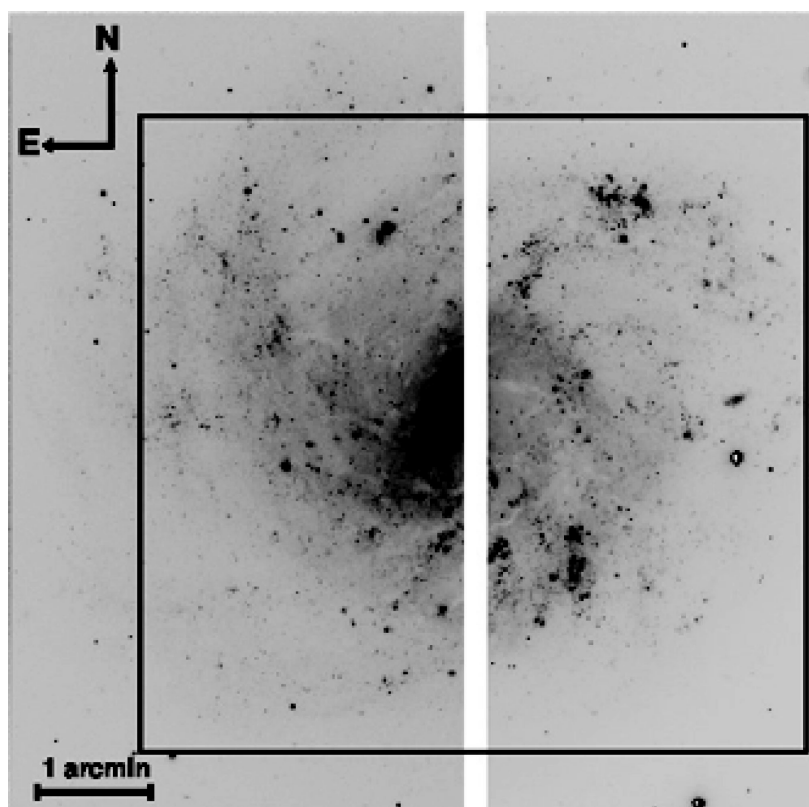

(a)

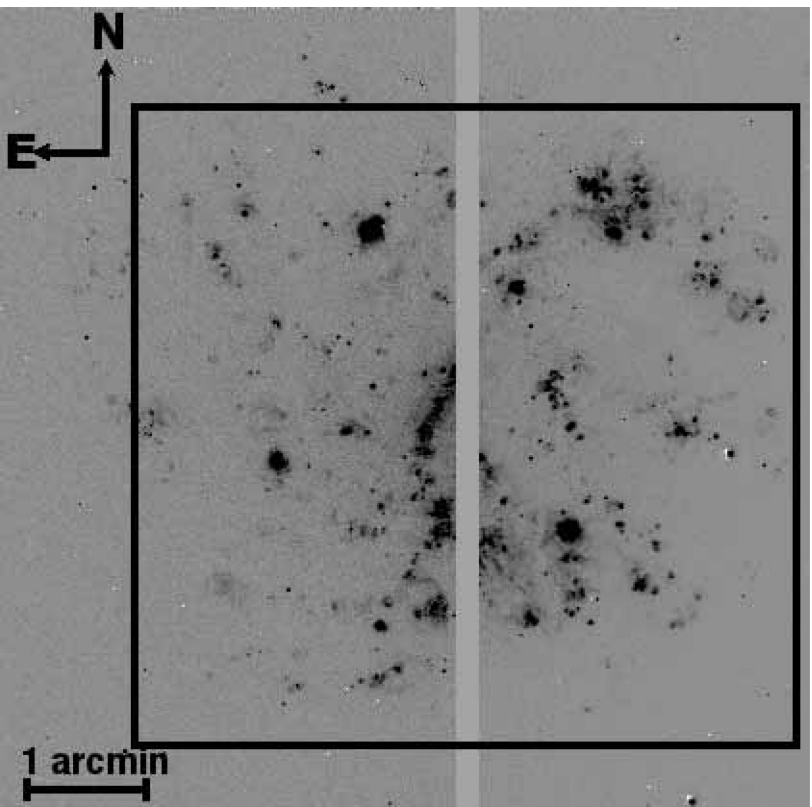

(b)

Figure 1. This figure shows (a) He II image and (b) the continuum subtracted $\mathrm{H} \alpha$ image of NGC 5068 obtained using a single pointing of $6.8 \times 6.8$ arcmin ${ }^{2}$ with the VLT/FORS1 with the smaller $5.5 \times 5.5 \operatorname{arcmin}^{2}$ Gemini/GMOS field of view indicated by the black square. The gap in the middle of the images represents the FORS1 chip gap hence we have no information about sources within this region.

for each filter while a single exposure of $250 \mathrm{~s}$ was used for additional narrow-band on- and off- $\mathrm{H} \alpha$ images $(\lambda 6563$ and $\lambda 6665$, respectively). Broad $V$ - and $B$-band high-throughput images of $250 \mathrm{~s}$ each were also acquired on the same night to supplement the narrow-band images. The field of view of the FORS1 images is presented in Fig. 1, the black square indicates the region covered by our spectroscopic observations which will be discussed further in Section 2.3.

Data reduction, including bias subtraction, flat-fielding and image combining were achieved using standard procedures in IRAF (Tody 1986). Photometry was performed using the DAOPHOT routine for the $B, V, \lambda 4684$ and $\lambda 4781$ images. Individual sources were fitted with a Gaussian point spread function (PSF) to determine their magnitude and its associated error.

Fig. 2 shows the log distribution of the number of sources within each 0.4-mag bin. Following Bibby \& Crowther (2010) we fit a power law to the bright end of the distribution using IDL to assess the completeness of our imaging. The 100 per cent completeness limit is indicated by the turnover of the distribution where the observed data deviate from the extrapolated power law. The 100 per cent completeness limit for our NGC 5068 narrow-band imaging corresponds to $m_{4684}=24.0 \mathrm{mag}$. We define the 50 per cent completeness limit as the magnitude at which only 50 per cent of the sources predicted by the power law are detected, finding a 50 per cent detection limit of $m_{4686}=24.8 \mathrm{mag}$ for NGC 5068. The significance of these detection limits is discussed further in Section 6.2. The detection limits for the broad-band images are almost identical to those of the narrow-band imaging.

Typical photometric errors for all images were approximately \pm 0.04 mag for bright $\left(m_{4684}=21 \mathrm{mag}\right)$ with significantly higher errors of \pm 0.20 mag for the faintest stars $\left(m_{4684}=26 \mathrm{mag}\right)$ and $\pm 0.5 \mathrm{mag}$ for $m_{4684}=27 \mathrm{mag}$. Fig. 3 shows the distribution photometric errors for the $\lambda 4684$ images. In some instances, irrespective of the brightness of the source, severe crowding makes a PSF fit inappropriate, so no photometry is derived for some sources.

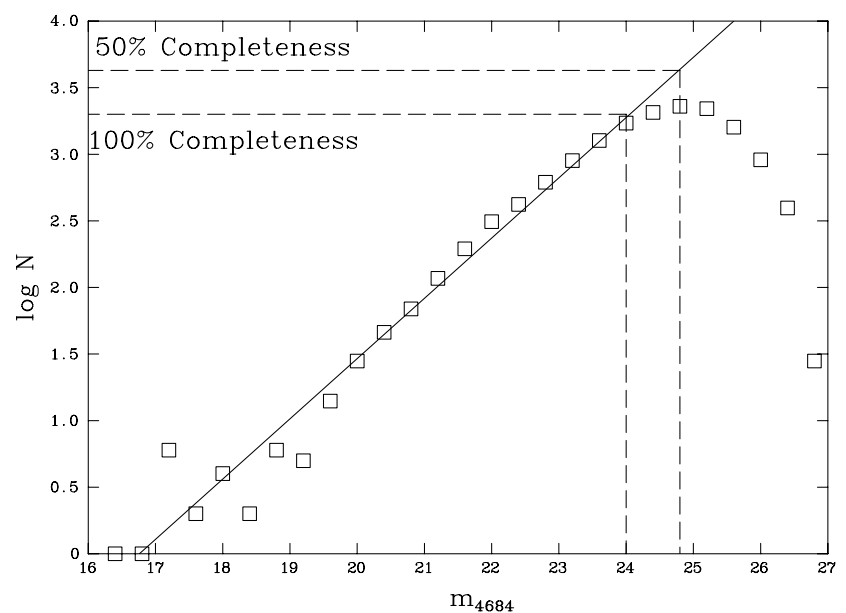

Figure 2. Plot showing how the distribution of photometric sources in NGC 5068 varies with apparent $\lambda 4684$ magnitude using 0.4 mag bins. The 100 and 50 per cent completeness limits are derived from this plot using the solid line which represents a linear fit to the brightest sources.

Absolute calibration for the broad-band images was achieved from observations of five stars within the standard star field SA 110-362 (Stetson 2000). For the narrow-band images the spectrophotometric standard star LTT 7987 (Hamuy et al. 1994) was used to obtain photometric zero-points since standard star fields are not available. The associated error on the calculated zero-points is \pm 0.15 mag.

\subsection{Source selection}

The $\lambda 4684$ narrow-band filter is coincident with the $\mathrm{N}$ III $\lambda 4640$, He II $\lambda 4686$ and $\mathrm{C}_{\text {IV }} \lambda 4650 \mathrm{WR}$ emission lines and the $\lambda 4781$ filter is placed on the continuum where there are no WR lines present. 


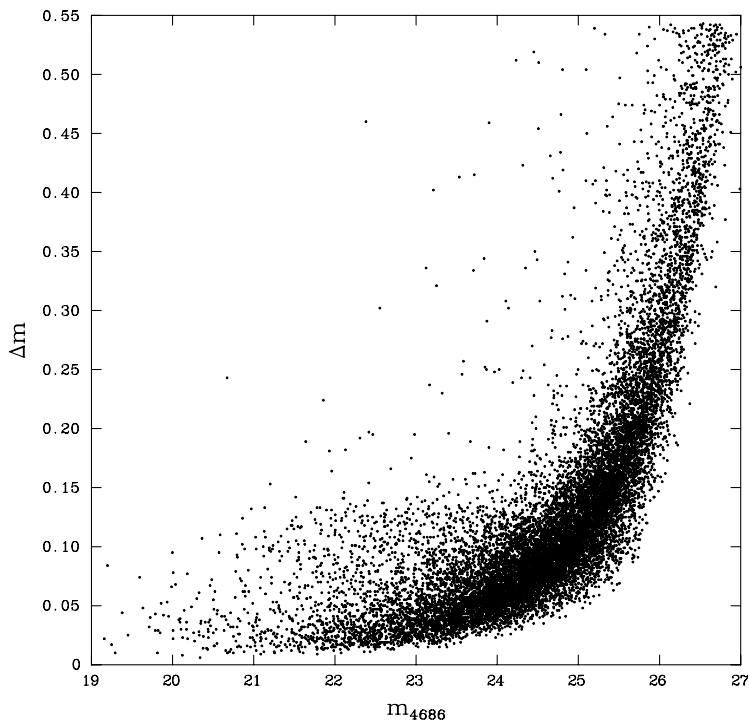

Figure 3. Photometric errors as a function of apparent magnitude for all sources detected in the $\lambda 4684$ VLT/FORS1 image of NGC 5068.

The 'blinking' technique, pioneered by Moffat \& Shara (1983), is the easiest way of identifying WR candidates and compares the $\lambda 4684, \lambda 4781$ and continuum subtracted image ( $\lambda 4684$ minus the $\lambda 4781$ image) to reveal emission-line stars.

In total 160 WR candidates were identified in NGC 5068, which are listed in Table 2. Photometry was obtained for 95 per cent of the candidates in at least the $\lambda 4684$ filter. The few sources for which $\lambda 4684$ photometry was not obtained were either too faint, extended or were located in a crowded region which makes reliable photometry difficult. Very extended or crowded sources are noted in Table 2, as are three sources which lay at the very edge of the FORS1 CCD that naturally represent less secure candidates. Although all 160 candidates were identified from the 'blinking' technique, photometric errors indicates that only 59 sources are formally $3 \sigma$ detections.

To enable accurate mapping of the WR candidates an astrometric solution was achieved by comparing the measured position of several stars in the image to their positions found in the Guide Star Catalogue. We found offsets of -0.6 and +0.22 arcsec for the right ascension and declination, respectively, which have been applied to the WR candidates listed in Table 2. Finding charts for all the WR candidates are available in the online appendix (see Supporting Information), an example of which is shown in Fig. 4.

\subsection{MOS spectroscopy}

Multi-Object Spectroscopy (MOS) of WR candidates in NGC 5068 was obtained in 2009 March-April using the GMOS at the GeminiSouth telescope in Cerro Pachon, Chile.

Since the $\lambda 4684$ and $\lambda 4781$ narrow-band imaging were obtained from the VLT, ${ }^{1}$ broad-band ( $g^{\prime}$ filter) pre-imaging was obtained using GMOS to aid the MOS mask design. The field of view of GMOS is $5.5 \times 5.5 \operatorname{arcmin}^{2}$ compared to the $6.8 \times 6.8 \operatorname{arcmin}^{2}$ VLT field (Fig. 1), hence some of the WR candidates lay beyond the GMOS field. The GMOS field of view was chosen to maximize

\footnotetext{
${ }^{1} \lambda 4684$ and adjacent continuum filters were subsequently acquired for Gemini/GMOS
}

the number of WR candidates and is overlaid on the VLT field in Fig. 1; only six sources were outside the GMOS field.

Three MOS masks were designed using 0.75 -arcsec slits and the R150 grating with a dispersion of $\sim 3.5 \AA$ pixel $^{-1}$. The spectral coverage was typically from $\sim 3900$ to $9000 \AA$ to include all of the WR and nebular diagnostic lines. Data longward of $\sim 7000 \AA$ suffered from fringing effects and second-order contamination so were unreliable, however, this is not a concern as no diagnostic lines lie within this region.

For the first MOS mask (\#1) six 430-s exposures were obtained under good seeing conditions which over the duration of the observations was an average of 0.87 arcsec. WR candidates included in MOS mask \#1 had the brightest $\lambda 4684-\lambda 4781$ photometric excesses requiring a signal-to-noise ratio $(\mathrm{S} / \mathrm{N}) \sim 3-5$ for detection. MOS masks \#2 and \#3 had six longer exposures of $1400 \mathrm{~s}$ each, producing a higher $\mathrm{S} / \mathrm{N}$ of $\sim 10$ to detect the fainter WR candidates. Seeing conditions for MOS mask \#2 were similar to the mask \#1, although conditions deteriorated to 1.16 arcsec for mask \#3.

64 out of the 160 WR candidates were spectroscopically observed ( $\sim 40$ per cent), a similar percentage to the $3 \sigma$ photometrically significant sources ( 23 from 59 cases). To fill the gaps in the MOS mask design, four $\mathrm{H}$ II regions, identified from our $\mathrm{H} \alpha$ images were added, allowing nebular properties to be investigated. Additional targets were spectroscopically observed in regions of NGC 5068 where we were unable to identify suitable WR candidates due to the FORS chip gaps, two of which were spectroscopically observed.

Spectroscopic data were reduced and extracted using standard procedures within the Gemini IRAF package. Spectra were wavelength calibrated using observations of an internal $\mathrm{CuAr}$ arc lamp with each of the three MOS masks. Absolute flux calibrations were achieved from observations of the spectrophotometric standard star LTT 7987 (Hamuy et al. 1994) using the STARLINK package FIGARO.

In order to assess the reliability of our photometry we convolved the observed flux calibrated object spectra with the response function of the interference filter used in the narrow-band imaging to determine a spectroscopic magnitude for each source; a comparison of which is shown in Fig. 5. Most sources are in reasonable agreement, with the exception of source \#17, the $m_{\lambda 4684}-m_{\lambda 4781}$ excess of +1.12 is inconsistent with the WC subtype determined from spectroscopy (see Section 4.2). This source was included as a candidate following visual inspection of the continuum subtracted image which clearly showed the presence of WR emission, hence photometry for this source is unreliable.

\section{NEBULAR PROPERTIES}

In this section we use our GMOS spectroscopy to derive the nebular properties of $\mathrm{H}_{\text {II }}$ regions in NGC 5068, comparing our results with previous estimates. Approximately 30 per cent of our spectroscopy of WR candidates contained nebular lines in addition to the four $\mathrm{H}$ II regions that were specifically targeted for this purpose. We performed Gaussian fits to the excited nebular (and stellar) emission lines using the EMISSION LINE FITTING (ELF) routine within the STARLINK package DIPSO.

\subsection{Interstellar extinction}

The interstellar extinction for each source was determined from the observed Balmer line ratios of $F_{\mathrm{H} \alpha} / F_{\mathrm{H} \beta}$ with Case B recombination theory (Hummer \& Storey 1987), assuming $n_{\mathrm{e}} \sim 100 \mathrm{~cm}^{-3}, T_{\mathrm{e}} \sim$ $10^{4} \mathrm{~K}$ and a standard Galactic extinction law (Seaton 1979). 
Table 2. Catalogue of WR candidates in NGC 5608 ordered by right ascension. Absolute magnitudes are derived using a distance of $5.45 \mathrm{Mpc}$ (Herrmann et al. 2008). Where spectra have been obtained the derived $E(B-V)$ is used. For those sources with no nebular lines an average $E(B-V)=0.259$ mag is used. The spectral classification determined from spectroscopy is listed along with the sources association with a H II region. 'Faint' implies nebular-like H $\alpha$ emission, 'bright' implies a more point source $\mathrm{H} \alpha$ emission while those in the brightest $\mathrm{H}$ in regions in NGC 5068 are referred to using ID numbers in Table 6. The final column indicates what finding chart the source is located in Appendix A (see Supporting Information).

\begin{tabular}{|c|c|c|c|c|c|c|c|c|c|c|c|c|c|}
\hline ID & $\begin{array}{c}\text { RA } \\
(\mathrm{J} 2000)\end{array}$ & $\begin{array}{l}\text { Dec. } \\
(\mathrm{J} 2000)\end{array}$ & $r / R_{25}$ & $\begin{array}{c}m_{V} \\
(\mathrm{mag})\end{array}$ & $\begin{array}{c}m_{B}-m_{V} \\
\quad(\mathrm{mag})\end{array}$ & $\begin{array}{l}m_{4684} \\
(\mathrm{mag})\end{array}$ & $\begin{array}{c}m_{4684}-m_{4781} \\
(\mathrm{mag})\end{array}$ & $\begin{array}{c}E(B-V) \\
\quad(\mathrm{mag})\end{array}$ & $\begin{array}{c}M_{V} \\
\text { (mag) }\end{array}$ & $\begin{array}{l}M_{4684} \\
(\mathrm{mag})\end{array}$ & $\begin{array}{l}\text { Spectral } \\
\text { type }\end{array}$ & H II region & $\begin{array}{l}\text { Finding } \\
\text { chart }\end{array}$ \\
\hline 1 & $13: 18: 41.27$ & $-20: 59: 53.1$ & 1.20 & - & - & $24.82 \pm 0.09$ & $-0.72 \pm 0.15$ & 0.259 & - & -4.79 & $(\mathrm{WN})(1)$ & - & 28 \\
\hline 2 & 13:18:41.27 & $-21: 04: 36.9$ & 1.07 & $21.68 \pm 0.07$ & $+1.76 \pm 0.07$ & $22.82 \pm 0.02$ & $-0.16 \pm 0.10$ & 0.259 & -7.80 & -6.79 & (No WR) & - & 23 \\
\hline 4 & 13:18:43.30 & $-21: 03: 41.2$ & 0.81 & $21.71 \pm 0.02$ & $+1.72 \pm 0.02$ & $22.81 \pm 0.02$ & $-0.09 \pm 0.12$ & 0.259 & -7.77 & -6.80 & (No WR) & Faint & 23 \\
\hline 5 & $13: 18: 43.36$ & $-21: 01: 29.1$ & 0.80 & Extended & & & & 0.259 & & & (WR?) & Faint & 24 \\
\hline 6 & 13:18:43.44 & $-21: 01: 31.5$ & 0.79 & $23.76 \pm 0.26$ & $+0.17 \pm 0.26$ & $22.95 \pm 0.03$ & $-1.46 \pm 0.23$ & 0.247 & -5.69 & -6.62 & WN4-6(2) & Faint & 24 \\
\hline 9 & 13:18:44.41 & $-21: 02: 34.1$ & 0.66 & $21.75 \pm 0.45$ & $-0.08 \pm 0.45$ & $21.83 \pm 0.08$ & $-0.21 \pm 0.08$ & 0.259 & -7.73 & -7.78 & (No WR) & Bright & 22 \\
\hline 10 & $13: 18: 44.75$ & $-21: 01: 04.3$ & 0.78 & $24.30 \pm 0.09$ & $+0.64 \pm 0.11$ & - & - & 0.428 & -5.18 & - & WC4-5(1) & Faint & 25 \\
\hline 11 & 13:18:44.85 & $-21: 00: 50.4$ & 0.82 & $21.68 \pm 0.02$ & $+0.17 \pm 0.06$ & $22.09 \pm 0.07$ & $-0.09 \pm 0.09$ & 0.259 & -7.80 & -7.52 & (No WR) & $\mathrm{H}_{\text {II } \# 3}$ & 25 \\
\hline 12 & 13:18:45.09 & $-21: 01: 03.8$ & 0.76 & $22.81 \pm 0.06$ & $+0.16 \pm 0.07$ & $22.35 \pm 0.02$ & $-0.95 \pm 0.25$ & 0.284 & -6.75 & -7.35 & WCE(1) & Bright & 25 \\
\hline 13 & $13: 18: 45.23$ & $-21: 02: 25.6$ & 0.60 & $21.75 \pm 0.02$ & $+0.05 \pm 0.04$ & $21.90 \pm 0.03$ & $-0.09 \pm 0.10$ & 0.465 & -8.37 & -8.45 & WCE(1)(?) & Bright & 22 \\
\hline 18 & 13:18:46.12 & $-21: 03: 25.0$ & 0.62 & - & - & $24.59 \pm 0.05$ & $-0.55 \pm 0.09$ & 59 & - & -5.02 & $(\mathrm{WN})(1)$ & - & 20 \\
\hline 19 & 13:18:46.63 & $-21: 03: 32.0$ & 0.61 & $23.43 \pm 0.03$ & $+0.43 \pm 0.06$ & $23.43 \pm 0.04$ & $-0.63 \pm 0.11$ & 0.259 & -6.05 & -6.18 & WC(1) & Bright & 20 \\
\hline 20 & 13:18:46.96 & $-21: 01: 47.5$ & 0.54 & $23.74 \pm 0.11$ & $-0.13 \pm 0.11$ & $23.62 \pm 0.05$ & $-0.11 \pm 0.26$ & 0.259 & & -5.99 & (No WR) & - & 21 \\
\hline 21 & 13:18:47.01 & $-21: 00: 33.8$ & 0.77 & $21.72 \pm 0.21$ & $+0.89 \pm 0.21$ & $22.79 \pm 0.03$ & - & 0.595 & -8.80 & -8.03 & WNE(2)(?) & I II \#6 & 26 \\
\hline 22 & 13:18:47.09 & $-21: 00: 25.3$ & 0.80 & $23.15 \pm 0.45$ & $-0.01 \pm 0.45$ & - & - & & -6.33 & - & No WR & Bright & 27 \\
\hline 23 & 13:18:47.16 & $-21: 03: 54.0$ & 0.65 & $23.75 \pm 0.27$ & $-0.07 \pm 0.29$ & - & - & 59 & -5.73 & - & (WR?) & Н II \#7 & 20 \\
\hline 24 & $13: 18: 47.24$ & $-21: 00: 36.5$ & 0.75 & - & - & - & - & 0.259 & - & - & (WR?) & - & 26 \\
\hline 25 & $13: 18: 47.438$ & $-21: 00: 19.7$ & 0.81 & $20.29 \pm 0.10$ & $+0.12 \pm 0.11$ & $20.44 \pm 0.04$ & $-0.01 \pm 0.33$ & 259 & -9.19 & -9.17 & No WR & Bright & 27 \\
\hline 26 & 13:18:47.44 & $-21: 03: 53.2$ & 0.64 & $23.37 \pm 0.13$ & $-0.06 \pm 0.15$ & $23.71 \pm 0.06$ & $-0.22 \pm 0.50$ & & -6.11 & -5.90 & (No & I II \#7 & 20 \\
\hline 27 & 13:18:47.47 & $-21: 01: 57.4$ & 0.48 & $23.39 \pm 0.09$ & $+1.83 \pm 0.15$ & $24.52 \pm 0.09$ & $-0.38 \pm 0.09$ & 259 & -6.09 & -5.09 & (No WR) & - & 21 \\
\hline 28 & $13: 18: 47.50$ & $-21: 03: 43.2$ & 0.60 & $22.78 \pm 0.09$ & $+0.07 \pm 0.09$ & $22.72 \pm 0.01$ & $-0.18 \pm 0.02$ & 0.159 & -6.39 & -6.53 & $(\mathrm{WN})(1)$ & Faint & 20 \\
\hline 29 & 13:18:47.50 & $-21: 03: 28.3$ & 0.55 & $25.19 \pm 0.05$ & $+0.01 \pm 0.12$ & $24.80 \pm 0.11$ & $-0.30 \pm 0.31$ & 0.259 & -4.29 & -4.81 & (No & - & 20 \\
\hline 38 & $13: 18$ & -21 & 0.72 & - & - & - & - & & - & - & (WR?) & Faint & 26 \\
\hline 39 & 13:18:48.29 & $-21: 00: 33.4$ & 0.72 & $22.59 \pm$ & $+0.21 \pm$ & $22.75 \pm 0.05$ & $-0.04 \pm$ & 59 & -6.89 & -6.86 & $\mathrm{WN}(1)$ & - & 26 \\
\hline 40 & $13: 18: 48.56$ & $-21: 00: 17.3$ & 0.78 & $20.59 \pm 0.04$ & $+0.38 \pm 0.06$ & $21.23 \pm 0.05$ & $-0.03 \pm 0.37$ & 0.259 & -8.89 & -8.38 & (No WR) & Н II \#10 & 27 \\
\hline 41 & 13:18:48.98 & $-21: 00: 21.0$ & 0.74 & $19.42 \pm 0.06$ & $+0.27 \pm 0.06$ & $19.82 \pm$ & $-0.02 \pm$ & 0.259 & -10.06 & -9.79 & No WR & Н II \#11 & 27 \\
\hline 42 & 13:18:48.98 & $-21: 00: 25.4$ & 0.72 & $7 \pm$ & 99 & .06 & -0 . & & -8 & -6 & (WC & Faint & 27 \\
\hline 43 & 13:18:49.15 & $-21: 02: 33.1$ & 0.35 & $22.90 \pm$ & $+0.09 \pm 0.05$ & $22.42 \pm 0.02$ & $-0.65 \pm 0.06$ & 59 & -6 & -7.19 & WN5b(3) & - & 15 \\
\hline 44 & $13: 18: 49.31$ & $-21: 02: 00.2$ & 0.36 & $22.93 \pm 0.28$ & $-0.21 \pm 0.29$ & $23.00 \pm 0.10$ & $-0.30 \pm 0.10$ & 0.259 & -6.55 & -6.66 & $(\mathrm{WN})(1)$ & - & 15 \\
\hline 45 & 13:18:49.36 & $-21: 00: 21.6$ & 0.73 & $19.53 \pm$ & $+0.38 \pm 0.16$ & $17 \pm$ & \pm 0 & 259 & -9.95 & -9.44 & No WR & $\mathrm{H}_{\text {II }} \# 12$ & 27 \\
\hline 46 & 13:18:49.47 & $-21: 02: 34.5$ & 0.33 & $1.10 \pm($ & $07 \pm 0.12$ & $.31 \pm 0.03$ & $+0.02 \pm 0.06$ & 59 & -8 & -8.30 & (No WR) & Bright & 15 \\
\hline 47 & 13:18:49.49 & $-21: 03: 55.9$ & 0.57 & $21.56 \pm 0.21$ & $-0.10 \pm 0.22$ & $21.55 \pm 0.09$ & $-0.38 \pm 0.15$ & 259 & -7.92 & -8.06 & (No WR) & Faint & 19 \\
\hline 48 & $13: 18: 49.60$ & $-21: 03: 38.3$ & 0.49 & - & - & - & - & 0.259 & - & - & (WR?) & Faint & 19 \\
\hline 49 & $13: 18: 49.69$ & $-21: 03: 19.9$ & 0.42 & $20.05 \pm 0.07$ & $+0.31 \pm 0.07$ & $20.33 \pm 0.01$ & $-0.03 \pm 0.07$ & 0.259 & -9.43 & -9.28 & (No WR) & $\mathrm{H}_{\text {II }} \# 14$ & 29 \\
\hline 50 & 13:18:49.72 & $-21: 03: 41.7$ & 0.50 & $1.46 \pm 0$ & $-0.13 \pm 0.10$ & $21.32 \pm 0.09$ & $-0.27 \pm 0.09$ & 259 & -8.02 & -8.29 & $(\mathrm{WN})(2)$ & - & 19 \\
\hline 51 & 13:18:49.78 & $-21: 04: 06.8$ & 0.61 & $22.64 \pm 0.08$ & $-0.34 \pm 0.09$ & $22.68 \pm 0.03$ & $-0.59 \pm 0.49$ & 0.259 & -6.84 & -6.93 & $(\mathrm{WN})(1)$ & Bright & 19 \\
\hline 52 & $13: 18: 49.80$ & $-21: 02: 28.2$ & 0.30 & $22.38 \pm 0.14$ & $-1.78 \pm 0.15$ & $20.99 \pm 0.02$ & $-0.16 \pm 0.15$ & 0.259 & -7.10 & -8.62 & (No WR) & $\mathrm{H}_{\text {II }} \# 13$ & 15 \\
\hline 53 & $13: 18: 49.80$ & $-21: 02: 26.5$ & 0.30 & - & - & $23.14 \pm 0.09$ & $-0.35 \pm 0.09$ & 0.259 & - & -6.47 & $(\mathrm{WN})(1)$ & $\mathrm{H}_{\text {II }} \# 13$ & 15 \\
\hline 54 & 13:18:49.81 & $-21: 03: 48.7$ & 0.53 & $21.74 \pm 0.08$ & $+0.19 \pm 0.09$ & $21.92 \pm 0.05$ & $-0.02 \pm 0.54$ & 0.101 & -7.25 & -7.12 & No WR & - & 19 \\
\hline 55 & $13: 18: 49.84$ & $-21: 03: 20.7$ & 0.42 & $22.11 \pm 0.05$ & $+0.08 \pm 0.09$ & $22.43 \pm 0.05$ & $-0.23 \pm 0.15$ & 0.259 & -7.37 & -7.18 & No WR & $\mathrm{H}_{\text {II }} \# 14$ & 29 \\
\hline 56 & $13: 18: 49.90$ & $-21: 02: 30.3$ & 0.30 & $23.28 \pm 0.20$ & $-0.12 \pm 0.21$ & $22.32 \pm 0.02$ & $-2.00 \pm 0.37$ & 0.259 & -6.20 & -7.29 & $(\mathrm{WC})(1)$ & $\mathrm{H}_{\text {II }} \# 13$ & 15 \\
\hline
\end{tabular}


Table 2 - continued

\begin{tabular}{|c|c|c|c|c|c|c|c|c|c|c|c|c|c|}
\hline ID & $\begin{array}{c}\text { RA } \\
(\mathrm{J} 2000)\end{array}$ & $\begin{array}{c}\text { Dec. } \\
(\mathrm{J} 2000)\end{array}$ & $r / R_{25}$ & $\begin{array}{c}m_{V} \\
(\mathrm{mag})\end{array}$ & $\begin{array}{c}m_{B}-m_{V} \\
(\mathrm{mag})\end{array}$ & $\begin{array}{l}m_{4684} \\
(\mathrm{mag})\end{array}$ & $\begin{array}{c}m_{4684}-m_{4781} \\
(\mathrm{mag})\end{array}$ & $\begin{array}{c}E(B-V) \\
\quad(\mathrm{mag})\end{array}$ & $\begin{array}{c}M_{V} \\
(\mathrm{mag})\end{array}$ & $\begin{array}{l}M_{4684} \\
\text { (mag) }\end{array}$ & $\begin{array}{l}\text { Spectral } \\
\text { type }\end{array}$ & gion & $\begin{array}{c}\text { Finding } \\
\text { chart }\end{array}$ \\
\hline 57 & $13: 18: 49.98$ & $-21: 03: 56.8$ & 0.56 & $3.64 \pm 0.05$ & $-0.59 \pm 0.07$ & $23.47 \pm 0.06$ & $-0.72 \pm 0.06$ & 0.259 & -5.84 & -6.14 & $(\mathrm{WN})(1)$ & - & 19 \\
\hline 58 & $13: 18: 49.99$ & $-21: 03: 23.7$ & 0.42 & $19.42 \pm 0.14$ & $+0.05 \pm 0.15$ & $19.19 \pm 0.08$ & $-0.39 \pm 0.49$ & 0.259 & -10.06 & -10.42 & (No WR) & Н II \#14 & 29 \\
\hline 59 & $13: 18: 50.03$ & $-21: 02: 17.5$ & 0.29 & - & - & $22.14 \pm 0.01$ & $-1.62 \pm 0.35$ & 0.259 & - & -7.47 & WCpec?(?) & Bright & 15 \\
\hline 60 & $13: 18: 50.08$ & $-21: 03: 21.3$ & 0.41 & $20.12 \pm 0.05$ & $+0.10 \pm 0.08$ & $20.46 \pm 0.07$ & $-0.16 \pm 0.37$ & 0.451 & -9.96 & -9.84 & No WR & Н II \#14 & 29 \\
\hline 61 & $13: 18: 50.11$ & $-21: 03: 45.2$ & 0.50 & $21.86 \pm 0.09$ & $-0.01 \pm 0.10$ & $23.56 \pm 0.08$ & $-0.47 \pm 0.15$ & 0.259 & -7.62 & -6.05 & $(\mathrm{WN})(1)$ & - & 19 \\
\hline 62 & $13: 18: 50.26$ & $-21: 03: 20.0$ & 0.41 & $24.24 \pm 0.33$ & $-0.53 \pm 0.33$ & $24.29 \pm 0.06$ & $-0.29 \pm 0.08$ & 0.259 & -5.24 & -5.32 & $(\mathrm{WN})(1)$ & Bright & 29 \\
\hline 63 & $13: 18: 50.27$ & $-21: 00: 51.4$ & 0.56 & $21.06 \pm 0.08$ & $+1.52 \pm 0.09$ & $22.04 \pm 0.04$ & $-0.10 \pm 0.29$ & 0.259 & -8.42 & -7.57 & No WR & Faint & 13 \\
\hline 64 & $13: 18: 50.38$ & $-21: 02: 15.6$ & 0.27 & $24.39 \pm 0.14$ & $-0.18 \pm 0.15$ & $24.36 \pm 0.07$ & $-0.47 \pm 0.14$ & 0.259 & -5.09 & -5.25 & $(\mathrm{WN})(1)$ & Faint & 15 \\
\hline 65 & $13: 18: 50.40$ & $-21: 02: 18.6$ & 0.27 & $21.18 \pm 0.09$ & $+0.12 \pm 0.09$ & $20.95 \pm 0.01$ & $-0.54 \pm 0.14$ & 0.259 & -8.30 & -8.66 & WC6(2) & Bright & 15 \\
\hline 66 & $13: 18: 50.41$ & $-21: 03: 42.9$ & 0.48 & $24.56 \pm 0.12$ & $-0.42 \pm 0.14$ & $23.95 \pm 0.06$ & $-0.41 \pm 0.14$ & 0.259 & -4.92 & -5.66 & $(\mathrm{WN}$ & - & 19 \\
\hline 67 & $13: 18: 50.42$ & $-21: 02: 01.8$ & 0.29 & $22.15 \pm 0.52$ & $-0.26 \pm 0.53$ & $21.87 \pm 0.05$ & $-0.26 \pm 0.53$ & 59 & -7.33 & -7.74 & (No WR) & Bright & 15 \\
\hline 68 & $13: 18: 50.50$ & $-21: 02: 02.2$ & 0.28 & Blended with & A24 & & & 0.259 & & & (WR?) & Bright & 15 \\
\hline 69 & $13: 18: 50.66$ & $-21: 02: 06.2$ & 0.27 & - & - & $22.04 \pm 0.03$ & $-0.49 \pm 0.03$ & 0.259 & - & -7.57 & $(\mathrm{WN}$ & H II \#15 & 15 \\
\hline 70 & $13: 18: 50.67$ & $-21: 02: 07.6$ & 0.26 & $20.37 \pm 0.13$ & $+0.02 \pm 0.13$ & $20.32 \pm 0.03$ & $-0.10 \pm 0.11$ & & -9.11 & -9.29 & (No & $\mathrm{H}_{\text {II }} \# 15$ & 15 \\
\hline 71 & $13: 18: 50.80$ & $-21: 01: 08.6$ & 0.46 & $20.70 \pm 0.21$ & $+1.70 \pm 0.21$ & $21.79 \pm 0.04$ & $-0.09 \pm 0.09$ & 59 & -8.78 & -7.82 & No WR & Faint & 13 \\
\hline 72 & $13: 18: 51.05$ & $-21: 02: 22.4$ & 0.22 & - & - & - & - & 0.259 & - & - & (WR?) & - & 15 \\
\hline 73 & $13: 18: 51.16$ & $-21: 04: 09.3$ & 0.59 & $21.07 \pm 0.17$ & $+0.03 \pm 0.18$ & $21.11 \pm 0.10$ & $-0.11 \pm 0.37$ & 0.277 & -8.47 & -8.57 & No WR & H II \#16 & 18 \\
\hline 74 & $13: 18: 51.30$ & $-21: 03: 50.5$ & 0.49 & - & - & $23.04 \pm 0.05$ & $-0.17 \pm 0.07$ & 59 & - & -6.57 & (No & Bright & 18 \\
\hline 75 & $13: 18: 51.43$ & $-21: 04: 09.0$ & 0.58 & $21.78 \pm$ & $+0.08 \pm$ & $21.79 \pm 0.05$ & $-0.25 \pm 0.11$ & & -7.70 & & (No V & H II \#16 & 18 \\
\hline 76 & $13: 18: 51.50$ & $-21: 04: 08.3$ & 0.58 & $20.69 \pm 0.18$ & $+0.27 \pm 0.18$ & $21.02 \pm 0.04$ & $-0.20 \pm 0.11$ & 0.259 & -8.79 & -8.59 & (No WR) & Н II \#16 & 18 \\
\hline 77 & 13:18:51.66 & $-21: 00: 05.1$ & 0.74 & $20.41 \pm 0.16$ & $+1.36 \pm 0.17$ & $21.32 \pm 0.05$ & $+0.03 \pm 0.08$ & 0.259 & -9.07 & -8.29 & No WR & Faint & 12 \\
\hline 78 & $13: 18: 51.68$ & $-21: 01: 18.4$ & 0.38 & $22.70 \pm 0.27$ & $-0.23 \pm 0.28$ & $22.34 \pm 0.04$ & $-0.35 \pm 0$ & 0.259 & -6.78 & -7.27 & WN & $\mathrm{H}_{\text {II }} \# 17$ & 14 \\
\hline 79 & $13: 18: 51.79$ & $-21: 01: 06.5$ & 0.44 & - & - & $23.89 \pm 0.08$ & $-0.44 \pm 0.08$ & & - & & & - & 13 \\
\hline 80 & $13: 18: 51.78$ & $-21: 03: 37.3$ & 0.42 & $25.25 \pm 0.06$ & $-0.33 \pm 0.10$ & - & - & 59 & -4.23 & - & (WR?) & Faint & 17 \\
\hline 81 & $13: 18: 51.85$ & $-21: 00: 49.6$ & 0.52 & $20.54 \pm 0.09$ & $+0.24 \pm 0.10$ & $21.20 \pm 0.05$ & $+0.02 \pm 0.05$ & 0.259 & -8.94 & -8.41 & No WR & Н II \#18 & 13 \\
\hline 82 & $13: 18: 51.86$ & $-21: 01: 16.1$ & 0.39 & $19.74 \pm 0.18$ & $+0.05 \pm 0.18$ & $19.63 \pm 0.05$ & $-0.25 \pm 0.07$ & 0.259 & -9.74 & -9.98 & $(\mathrm{WN})(8)$ & $\mathrm{H}_{\text {II }} \# 17$ & 14 \\
\hline 83 & $13: 18: 51.90$ & $-21: 00: 53.9$ & 0.49 & $20.87 \pm 0.14$ & $+0.15 \pm 0.14$ & & $+0.02 \pm 0.08$ & & -8.61 & & No & Faint & 13 \\
\hline 84 & $13: 18: 51.98$ & $-21: 03: 41.2$ & 0.44 & $24.52 \pm 0.02$ & $-0.21 \pm 0.04$ & $22.72 \pm 0.02$ & $-2.35 \pm 0.23$ & 50 & -4.96 & -6.89 & WC4-5(1) & - & 17 \\
\hline 85 & $13: 18: 52.04$ & $-21: 01: 14.9$ & 0.39 & $21.80 \pm 0.06$ & $+0.35 \pm 0.07$ & $22.23 \pm 0.03$ & $+0.06 \pm 0.39$ & 0259 & -7.68 & -7.38 & No WR & H II \#17 & 14 \\
\hline 86 & $13: 18: 52.15$ & $-21: 01: 18.8$ & 0.37 & $23.02 \pm 0.19$ & $-0.31 \pm 0.20$ & $22.57 \pm 0.03$ & $-0.31 \pm 0.04$ & 0.259 & -6.46 & -7.04 & $(\mathrm{WN})(1)$ & Bright & 14 \\
\hline 87 & $13: 18: 52.16$ & $-21: 01: 14.2$ & 0.39 & - & - & - & - & 0.259 & - & - & (WR?) & Faint & 14 \\
\hline 88 & $13: 18: 52.19$ & $-21: 01: 21.7$ & 0.35 & $24.41 \pm 0.12$ & $+0.15 \pm 0.14$ & $23.03 \pm 0.03$ & $-2.80 \pm 0.06$ & 0.259 & -5.07 & -6.58 & $(\mathrm{WC})(1)$ & - & 14 \\
\hline 89 & $13: 18: 52.22$ & $-21: 04: 07.0$ & 0.56 & - & - & $22.42 \pm 0.03$ & $-2.54 \pm 0.13$ & 0.259 & - & -7.19 & $(\mathrm{~W}$ & - & 18 \\
\hline 90 & $13: 18: 52.28$ & $-21: 01: 25.4$ & 0.33 & $23.82 \pm 0.09$ & $-0.17 \pm 0.10$ & $22.03 \pm 0.03$ & $-2.23 \pm 0.06$ & 0.259 & -5.66 & -7.58 & WC4-5(2) & - & 14 \\
\hline 91 & $13: 18: 52.29$ & $-21: 01: 22.6$ & 0.34 & $24.41 \pm 0.12$ & $+0.15 \pm 0.14$ & $23.03 \pm 0.04$ & $-2.80 \pm 0.06$ & 0.259 & -5.07 & -6.58 & $(\mathrm{WC})(1)$ & Bright & 14 \\
\hline 92 & $13: 18: 52.39$ & $-21: 03: 43.5$ & 0.44 & $22.34 \pm 0.15$ & $+0.18 \pm 0.16$ & $22.55 \pm 0.05$ & $-0.79 \pm 0.17$ & 0.259 & -7.14 & -7.06 & $(\mathrm{WN})(2)$ & Faint & 14 \\
\hline 93 & $13: 18: 52.57$ & $-21: 00: 57.8$ & 46 & 19 & $-0.14 \pm 0.02$ & 21.3 & $-0.11 \pm 0.35$ & & -7.79 & -7.96 & & Brig & 13 \\
\hline 94 & $13: 18: 52.71$ & $-21: 03: 04.3$ & 0.25 & $5.14 \pm 0.08$ & $-0.71 \pm 0.09$ & $22.55 \pm 0.02$ & $-3.36 \pm 0.05$ & 0 . & -4.34 & -7.06 & WC7(1) & - & 16 \\
\hline 95 & $13: 18: 52.72$ & $-21: 03: 27.6$ & 0.36 & - & - & $21.99 \pm 0.09$ & $+0.12 \pm 0.28$ & 0.259 & - & -7.62 & No WR & Faint & 17 \\
\hline 96 & $13: 18: 52.95$ & $-21: 02: 54.3$ & 0.19 & $23.46 \pm 0.31$ & $-0.15 \pm 0.32$ & $22.39 \pm 0.02$ & $-1.20 \pm 0.19$ & 0.259 & -6.02 & -7.22 & $(\mathrm{WN})(2)$ & Bright & 16 \\
\hline 97 & $13: 18: 53.06$ & $-21: 03: 56.8$ & 0.50 & $21.44 \pm 0.29$ & $+1.98 \pm 0.29$ & $22.64 \pm 0.04$ & $-0.12 \pm 0.09$ & & -8.04 & -0.91 & (INO & & 18 \\
\hline 98 & 13:18:53.06 & $-21: 03: 25.7$ & 0.34 & - & - & & - & 0. & - & - & (WR?) & Bright & 17 \\
\hline 99 & 13:18:53.08 & $-21: 03: 22.7$ & 0.33 & $23.66 \pm 0.04$ & $+0.53 \pm 0.05$ & - & - & 0.259 & -5.82 & - & WN6(3) & Bright & 17 \\
\hline 100 & $13: 18: 53.11$ & $-21: 03: 30.9$ & 0.37 & $23.17 \pm 0.33$ & $+0.12 \pm 0.33$ & $23.11 \pm 0.05$ & $-0.21 \pm 0.07$ & 0.259 & -6.31 & -6.50 & $(\mathrm{WN})(1)$ & Bright & 17 \\
\hline 101 & $13: 18: 53.13$ & $-21: 03: 36.6$ & 40 & 05 & $+0.21 \pm 0.06$ & $19.94 \pm$ & $+0.07=$ & & -9.79 & -9.67 & & & 17 \\
\hline 102 & $13: 18: 53.14$ & $-21: 00: 32.2$ & 0.57 & $22.03 \pm 0.22$ & $+0.23 \pm 0.23$ & $22.38 \pm 0.06$ & $-0.13 \pm 0.39$ & 0 & -7.45 & -7.23 & (No WR) & Bright & 10 \\
\hline 103 & $13: 18: 53.23$ & $-21: 02: 43.5$ & 0.14 & $22.39 \pm 0.09$ & $-0.17 \pm 0.11$ & $22.36 \pm 0.02$ & $-0.18 \pm 0.45$ & 0.027 & -6.37 & -6.42 & No WR & Н II \#19 & 16 \\
\hline 104 & $13: 18: 54.15$ & $-21: 02: 03.8$ & 0.09 & Edge of chip & - & - & - & 0.147 & - & - & WN(1) & - & 8 \\
\hline 105 & $13: 18: 54.15$ & $-21: 03: 26.7$ & 0.34 & Edge of chip & - & - & - & - & - & - & No WR & Bright & 7 \\
\hline 106 & $13: 18: 54.18$ & $-21: 00: 27.9$ & 0.59 & $23.21 \pm 0.12$ & $-0.19 \pm 0.13$ & $22.80 \pm 0.04$ & $-0.57 \pm 0.51$ & 0.087 & -5.74 & -6.19 & WN7(1) & Bright & 10 \\
\hline 107 & $13: 18: 54.19$ & $-21: 03: 07.6$ & 0.24 & Edge of chip & - & - & - & 0.259 & - & - & (WR?) & Bright & 7 \\
\hline 108 & $13: 18: 54.22$ & $-21: 00: 32.1$ & 0.56 & $20.88 \pm 0.05$ & $+0.01 \pm 0.05$ & $20.99 \pm 0.03$ & $-0.05 \pm 0.18$ & 0.259 & -8.60 & -8.62 & No WR & Faint & 10 \\
\hline 109 & $13: 18: 54.24$ & $-21: 02: 04.6$ & 0.08 & $20.65 \pm 0.26$ & $+0.57 \pm 0.27$ & $20.85 \pm 0.10$ & $-0.25 \pm 0.16$ & 0.259 & -8.83 & -8.7 & (No WR) & Bright & 8 \\
\hline 110 & $13: 18: 54.25$ & $-21: 03: 06.5$ & 0.24 & $24.40 \pm 0.03$ & $-0.28 \pm 0.10$ & $21.15 \pm 0.02$ & $-0.15 \pm 0.03$ & 0.102 & -5.08 & -8.46 & (No WR) & Faint & 7 \\
\hline 111 & $13: 18: 54.27$ & $-21: 02: 55.1$ & 0.18 & $25.04 \pm 0.12$ & $-0.57 \pm 0.14$ & $23.42 \pm 0.03$ & $-1.50 \pm 0.06$ & 0.259 & -4.44 & -6.19 & WNL(1) & - & 7 \\
\hline 112 & $13: 18: 54.31$ & $-21: 03: 08.2$ & 0.25 & - & - & $22.14 \pm 0.04$ & - & 0.102 & - & -6.91 & WC(1) & Faint & 7 \\
\hline 113 & $13: 18: 54.38$ & $-21: 00: 29.3$ & 0.58 & - & - & - & - & 0.259 & - & - & (WR?) & Faint & 10 \\
\hline 114 & $13: 18: 54.49$ & $-21: 02: 13.3$ & 0.04 & Crowded & & & & & & & (WR?) & Bright & 8 \\
\hline 115 & $13: 18: 54.60$ & $-21: 02: 07.9$ & 0.07 & $19.87 \pm 0.05$ & $+0.15 \pm 0.08$ & $19.99 \pm 0.09$ & $-0.03 \pm 0.21$ & 0.259 & -9.61 & -9.62 & No WR & Faint & 8 \\
\hline 116 & $13: 18: 54.67$ & $-21: 03: 10.8$ & 0.26 & $18.91 \pm 0.13$ & $+0.05 \pm 0.14$ & $18.99 \pm 0.06$ & $-0.16 \pm 0.44$ & 0.259 & -10.57 & -10.62 & No WR & Н II \#20 & 7 \\
\hline
\end{tabular}


Table 2 - continued

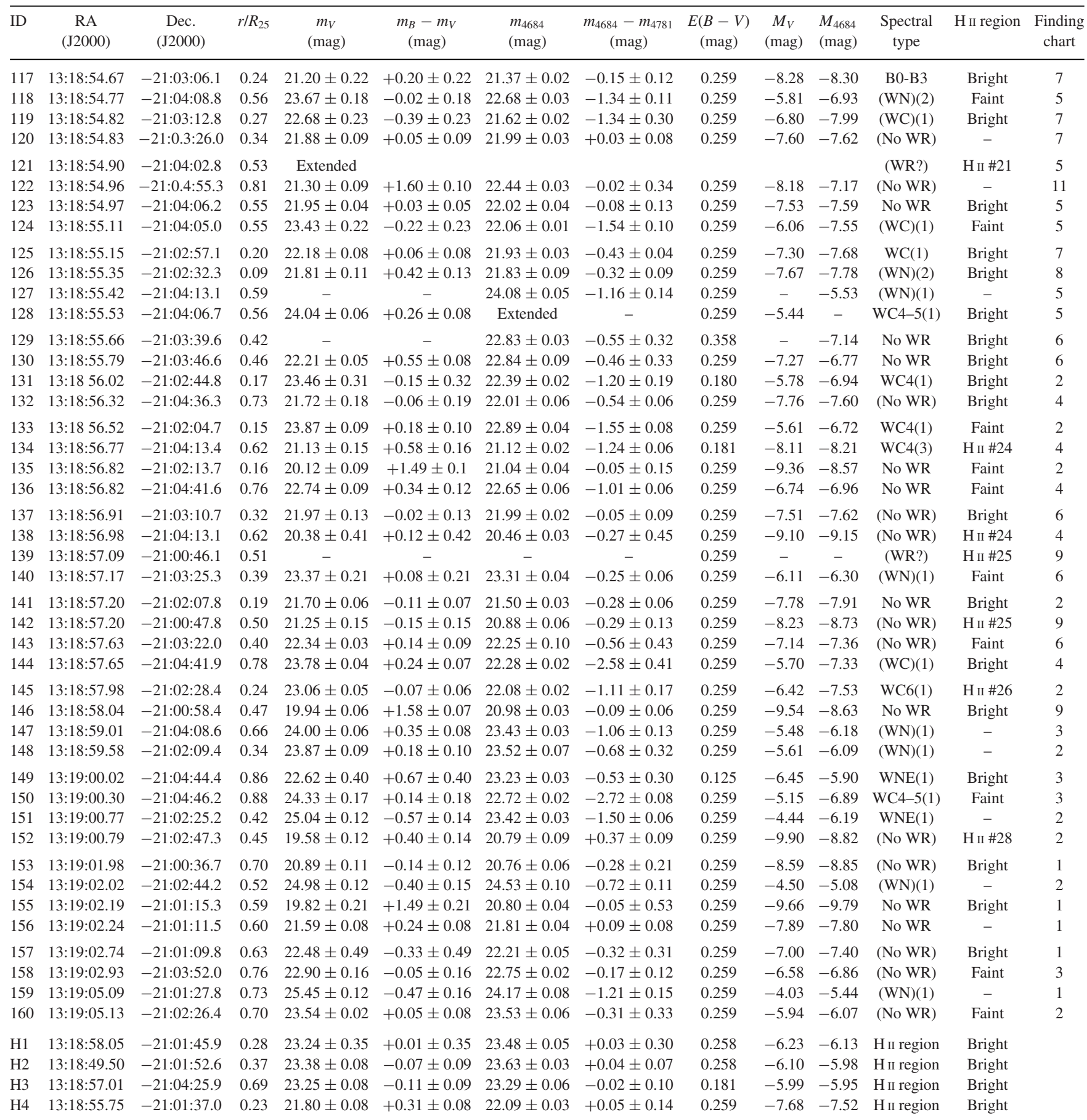

Individual values of $E(B-V)$ are listed for each source in Table 2 and range from just above the foreground extinction at $E(B-V)=$ 0.087 to $0.428 \mathrm{mag}$. The average extinction of $E(B-V)=0.259 \pm$ $0.035 \mathrm{mag}$ is applied to all sources for which no spectroscopic value can be derived. Taking into account the underlying Balmer absorption we estimate that the $\mathrm{H} \beta$ flux is underestimated by $1-13$ per cent based on measured equivalent widths of 15-300 A. Ryder (1995) derive similar values of extinction ranging from foreground extinction of $E(B-V)=0.007$ to $0.623 \mathrm{mag}$. The dereddened emission-line spectrum of the $\mathrm{H}$ II regions \#202 is shown as an example in Fig. 6 with all the nebular diagnostic lines indicated.

\subsection{Metallicity of NGC 5068}

Nebular emission lines from $\mathrm{H}$ II regions allow metallicity calculations for NGC 5068. Previously, Ryder (1995) used a combination of long-slit spectroscopy and multi-aperture plate slitlets to obtain spectra of $20 \mathrm{H}$ II regions within NGC 5068. Using the $R_{23}$ method (Pagel \& Edmunds 1981) they determined a metallicity gradient of $\log (\mathrm{O} / \mathrm{H})+12=(8.96 \pm 0.12)-(0.35 \pm 0.26)\left(r / R_{25}\right)$, where $r / R_{25}$ is the deprojected distance from the centre of the galaxy based on $R_{25}=3.62$ arcmin for NGC 5068 from de Vaucouleurs et al. (1991). Pilyugin, Vilchez \& Contini (2004) subsequently combined 


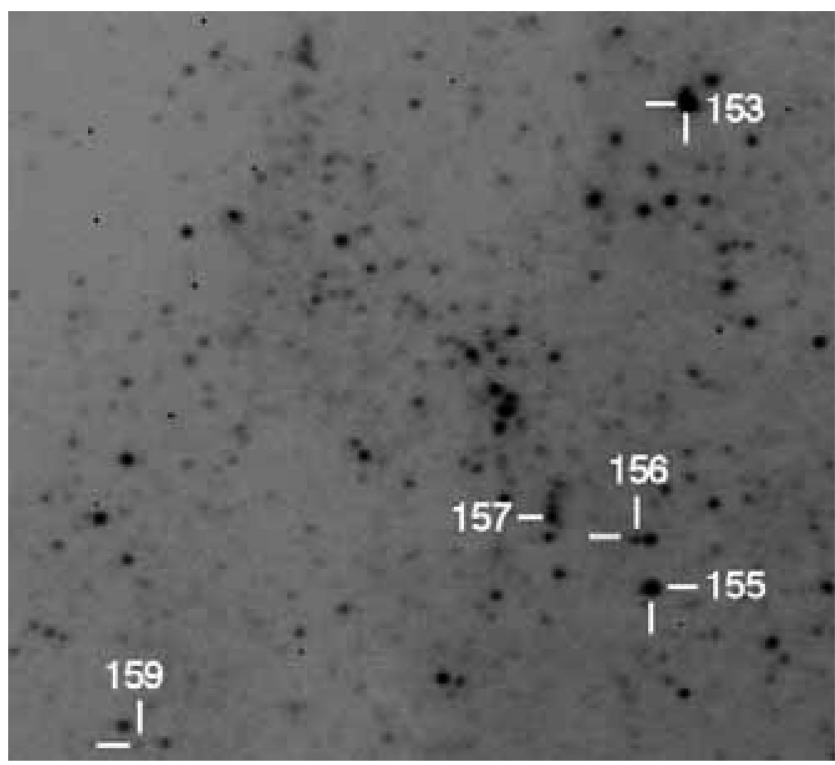

Figure 4. Finding chart A.2 showing the location of five sources with their ID number corresponding to that in Table 2. A key showing the location of each finding chart on the galaxy is also provided in Appendix A (see Supporting Information), and the finding chart for each source is listed in Table 2.

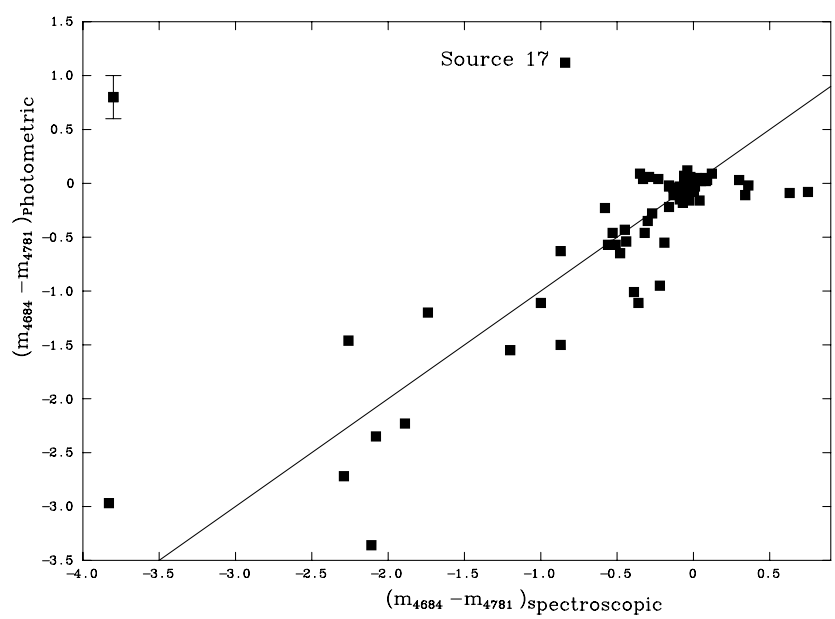

Figure 5. Comparison of spectroscopic and photometric $\lambda 4684$ excesses of NGC 5068 sources. The solid line indicated where the excesses are in agreement. The error bar $( \pm 0.2 \mathrm{mag})$ shown is typical of faint sources $(\sim 24$ $26 \mathrm{mag}$ ), however, errors are lower for brighter sources.

the spectroscopic observations by Ryder (1995) with those from McCall, Rybski \& Shields (1985) and recalculated a metallicity gradient of $\log (\mathrm{O} / \mathrm{H})+12=8.32+0.08\left(r / R_{25}\right)$ using the excitation parameter, $P$, rather than from empirical values. This result suggests that the centre of the galaxy is more metal poor than the outer disc. Only four instances of positive gradients were found by Pilyugin et al. (2004) from a sample of 54 galaxies. Usually, spiral galaxies have flat or negative metallicity gradients (Zaritsky, Kennicutt \& Huchra 1994), e.g. NGC 7793 (Bibby \& Crowther 2010) and M101 (Cedres, Urbaneja \& Cepa 2004).

Unfortunately, due to the low $\mathrm{S} / \mathrm{N}$ of our spectroscopic observations the $\lambda 4363 \AA[\mathrm{O}$ III] line is not detected so an accurate temperature, and hence emissivities cannot be calculated. Consequently we are unable to use the weak-lined methods described by

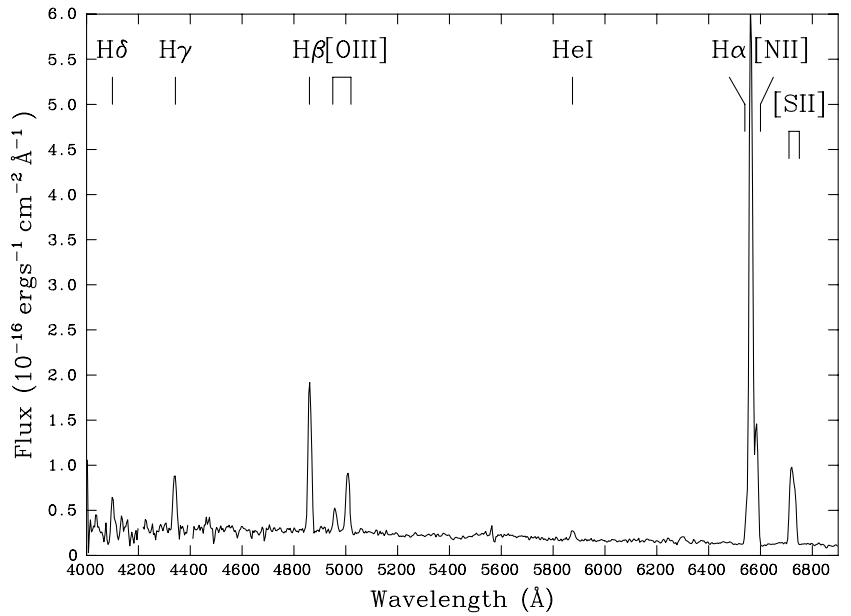

Figure 6. The dereddened GMOS spectra of the source \#202, a $\mathrm{H}$ II region within NGC 5068. The emission lines used for nebular analysis are indicated. The extinction determined from this spectrum is $E(B-V)=0.258 \mathrm{mag}$, close to the average extinction.

Osterbrock (1989) to determine the ionic abundances of $\mathrm{H}_{\text {II }}$ regions in NGC 5068; consequently we revert to strong-line methods described by Pettini \& Pagel (2004). In addition to the four H II regions included in our MOS masks, the $\mathrm{H} \alpha,[\mathrm{N}$ II], $\mathrm{H} \beta$ and [O III] lines required are present in $17 \mathrm{WR}$ spectra. We calculated the $[\mathrm{N} I] / \mathrm{H} \alpha$ (or $\mathrm{N} 2$ ) and $\left(\left[\mathrm{O}{ }_{\mathrm{III}}\right] / \mathrm{H} \beta\right) / \mathrm{N} 2$ (or $\mathrm{O} 3 \mathrm{~N} 2$ ) line ratios by fitting the nebular emission lines with a Gaussian profile using the ELF routine. The linear relationships of $\mathrm{N} 2$ and $\mathrm{O} 3 \mathrm{~N} 2$ with metallicity from Pettini \& Pagel (2004) were used to determine the metallicity of the $\mathrm{H}$ II region, shown in Table 3 . Using the gradient function in IDL we fit the average metallicities of the $\mathrm{H}_{\text {II }}$ regions and found a metallicity gradient of

$\log \frac{\mathrm{O}}{\mathrm{H}}+12=(8.74 \pm 0.15)-(0.61 \pm 0.22) \frac{r}{R_{25}}$

for NGC 5068 which is shown in Fig. 7. Metallicities range from $\log (\mathrm{O} / \mathrm{H})+12=8.10$ to 8.72 with a central metallicity of $\log (\mathrm{O} / \mathrm{H})+12=8.74$. The central metallicity is lower than that found by Ryder (1995), however, the metallicity gradient is consistent within errors indicating that different methods used $\left(R_{23}\right.$ versus $\mathrm{N} 2$ and O3N2) to derive metallicity may have a systematic offset (Kewley \& Dopita 2002; Kewley \& Ellison 2008). Unfortunately our spectral range does not include the [O II] $\lambda 3727 \AA$ line required for the $R_{23}$ method, so a full comparison of the two methods cannot be made. Finally, our negative metallicity gradient is inconsistent with the positive gradient found by Pilyugin et al. (2004) and their estimate of the central metallicity is $\sim 0.4$ dex lower than our own.

\section{WOLF-RAYET POPULATION}

We have identified broad WR emission features in 30 of the 64 sources spectroscopically observed, i.e. only 47 per cent of these candidates are confirmed. Note that these statistics exclude three candidates for which slits were misaligned, as a result of conversion between the FORS $1 \lambda 4684$ imaging to the $g^{\prime}$ GMOS pre-imaging for the MOS mask design.

Of the remaining spectroscopically observed candidates lacking WR emission features, one is an early B-type star while the others show no stellar features at the low $\mathrm{S} / \mathrm{N}$ of the observations. Photometrically, the majority of these sources indicated negligible or weak He II $\lambda 4686$ excesses. Indeed, only one (\#136) is a firm 
Table 3. $\mathrm{N} 2$ and $\mathrm{O} 3 \mathrm{~N} 2$ line ratios and corresponding metallicities for nebular sources in NGC 5068. The errors on $\log (\mathrm{O} / \mathrm{H})+12$ are dominated by the error on the fits to N2 $( \pm 0.41)$ and O3N2 $( \pm 0.25)$ from Pettini \& Pagel (2004). This corresponds to a error on the mean $\log (\mathrm{O} / \mathrm{H})+12$ of \pm 0.24 , and on the average $\log (\mathrm{O} / \mathrm{H})+12$ of \pm 0.17 .

\begin{tabular}{ccccccc}
\hline $\begin{array}{c}\text { Source } \\
\text { ID }\end{array}$ & $r / R_{25}$ & $\begin{array}{c}I([\mathrm{~N} \mathrm{II}]) / \\
I(\mathrm{H} \alpha)\end{array}$ & $\begin{array}{c}\log (\mathrm{O} / \mathrm{H}) \\
+12^{a}\end{array}$ & $\begin{array}{c}I([\mathrm{O} \text { III }]) / \\
I(\mathrm{H} \beta)\end{array}$ & $\begin{array}{c}\log (\mathrm{O} / \mathrm{H}) \\
+12^{b}\end{array}$ & $\begin{array}{c}\log (\mathrm{O} / \mathrm{H}) \\
+12_{\text {mean }}\end{array}$ \\
\hline 10 & 0.78 & 0.07 & 8.26 & 1.53 & 8.27 & 8.27 \\
12 & 0.76 & 0.10 & 8.32 & 1.40 & 8.39 & 8.34 \\
21 & 0.77 & 0.07 & 8.25 & 7.06 & 8.13 & 8.19 \\
45 & 0.73 & 0.07 & 8.24 & 1.13 & 8.38 & 8.31 \\
$\mathrm{H} 2$ & 0.37 & 0.13 & 8.39 & 0.47 & 8.58 & 8.49 \\
54 & 0.53 & 0.15 & 8.42 & - & - & 8.42 \\
55 & 0.42 & 0.13 & 8.40 & 0.90 & 8.50 & 8.45 \\
60 & 0.41 & 0.12 & 8.37 & 0.26 & 8.65 & 8.51 \\
73 & 0.59 & 0.10 & 8.32 & 0.49 & 8.54 & 8.43 \\
99 & 0.33 & 0.44 & 8.70 & 0.93 & 8.47 & 8.58 \\
103 & 0.14 & 0.36 & 8.65 & 0.29 & 8.79 & 8.72 \\
104 & 0.09 & 0.37 & 8.65 & 0.19 & 8.43 & 8.54 \\
128 & 0.56 & 0.09 & 8.31 & 1.63 & 8.27 & 8.29 \\
129 & 0.42 & 0.20 & 8.50 & 3.22 & 8.38 & 8.44 \\
130 & 0.46 & 0.17 & 8.47 & 0.62 & 8.58 & 8.53 \\
131 & 0.17 & 0.26 & 8.56 & 0.55 & 8.66 & 8.61 \\
H3 & 0.69 & 0.14 & 8.41 & 0.22 & 8.70 & 8.56 \\
145 & 0.24 & 0.19 & 8.49 & - & - & 8.49 \\
H1 & 0.28 & 0.21 & 8.51 & 0.31 & 8.66 & 8.59 \\
149 & 0.86 & 0.04 & 8.10 & 4.20 & 8.11 & 8.10 \\
150 & 0.88 & 0.07 & 8.23 & 2.71 & 8.25 & 8.24 \\
Average & & 0.155 & & & & $8.44 \pm 0.17$ \\
\hline
\end{tabular}

${ }^{a} \mathrm{~N} 2$.

${ }^{b} \mathrm{O} 3 \mathrm{~N} 2$.

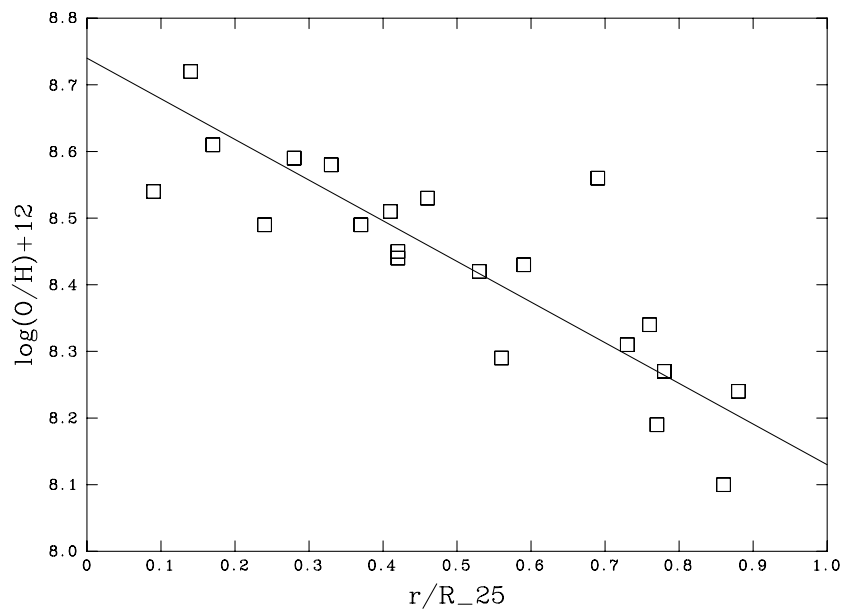

Figure 7. Deprojected distance from the centre of the galaxy versus the metallicity derived for $21 \mathrm{H}$ II regions in NGC 5068. The error on the individual points is \pm 0.19 .

WR candidate with a $\lambda 4684$ excess of $\geq 3 \sigma$, such that 21 of the 22 statistically significant sources were confirmed as WR stars.

Emission-line fluxes of $\mathrm{He}$ II $\lambda 4686+\mathrm{N}$ III-V $\lambda 4603-4641$ for WN stars and C III $\lambda 4647-4651+C_{\text {IV }} \lambda 5808$ for WC stars are fitted with Gaussian profiles using the ELF suite within the DIPSO package. Spectral classification systems by Smith, Shara \& Moffat (1996) and Crowther, De Marco \& Barlow (1998) are used to assign either a WN or WC subtype, respectively. At a distance of $5.45 \mathrm{Mpc}$ we cannot spatially resolve individual WR stars. We therefore adopt the luminosity calibrations derived by Crowther \& Hadfield (2006), which are based on average line luminosities for single WR stars in the LMC, to determine the number of $\mathrm{WN}$ and $\mathrm{WC}$ stars in each source. LMC templates are used in view of the metallicity of NGC 5068 obtained in Section 3. Table 4 records the line fluxes and luminosities for each WR source, the classification assigned and the number of WR stars present in that region.

\subsection{WN stars}

12 of the 30 sources confirmed reveal the characteristic signature of WN stars. If $\mathrm{N} \mathrm{V-III} \lambda 4603-4641$ is detected then its strength relative to the $\mathrm{He}$ II $\lambda 4686$ emission line is used to assign a refined classification of WN6 or WN7. For those WN stars where N v-III $\lambda 4603-4641$ was not detected we assume a WNE subtype. Midtype WN4-6 classifications are used when both He II $\lambda 4686$ and He II $\lambda 5411$ are detected. In one case we classify the WR source as WN5b since the WR emission features are much broader than typical WR stars. In total, we identify $18 \mathrm{WN}$ stars in the 12 sources. Fig. 8(a) shows the observed WN spectra of source \#99, which hosts two WN5-6 stars. The template spectra, shown by the dashed line, are taken from Crowther \& Hadfield (2006). The He II $\lambda 4686, \lambda 5411$ and $\mathrm{N}_{\text {III }} \lambda 4641$ WR emission lines are marked while the additional narrow lines are nebular emission lines.

\subsection{WC stars}

We identify strong blue and red emission WC features for 18 of the 30 sources spectroscopically confirmed as WR stars. Following the classification of Crowther et al. (1998) we use the C III $\lambda 5696 / C$ IV $\lambda 5808$ ratio to refine the subtype of the WC stars. We find that WC4-5 stars dominate the WC population, with no $\mathrm{C}_{\text {III }} \lambda 5696$ detected in the stellar spectrum, as is the case in other relatively metal-poor galaxies. A typical example is presented in Fig. 8(b) together with an LMC WC4 template (Crowther \& Hadfield 2006). These are used to determine the number of WC stars in each region, with most regions hosting a single WC star. In total, we identify 24 WC stars in the 18 sources exhibiting WC emission.

In addition to the 30 confirmed WR sources, broad emission at $\lambda 4660$ is seen in source $\# 59$, which we attribute to either $C_{\text {III }} \lambda 4650$, $\mathrm{C}_{\text {IV }} \lambda 4660$ or He II $\lambda 4686$, as indicated in Fig. 9. However, neither $\mathrm{C}_{\text {IV }} \lambda 5808$ nor $\mathrm{C}_{\text {III }} \lambda 5696$ is observed, from which we assign a WC?pec spectral type, but do not attempt to quantify the number of WR stars.

\section{H $\alpha$ IMAGING}

We have obtained narrow-band $\lambda 6563(\mathrm{H} \alpha)$ and adjacent continuum images using VLT/FORS1 to derive the total O star population and SFR of NGC 5068. Images were absolutely flux calibrated using the spectrophotometric standard star LTT 7987 (Hamuy et al. 1994) obtained on the same night as the science observations. The $\lambda 6563$ observations are contaminated by the $[\mathrm{N}$ II $] \lambda \lambda 6548,6583$ doublet which can account for as much as $\sim 30$ per cent of the total emission in metal-rich regions. Our spectroscopic observations of $\mathrm{H}$ II regions resolve the $\mathrm{H} \alpha$ and $\left[\mathrm{N}\right.$ II] lines finding on average $\left[\mathrm{N}_{\mathrm{II}}\right] / \mathrm{H} \alpha=0.155$ (Table 3 which we applied to our measured $\lambda 6563$ fluxes). We corrected the $\mathrm{H} \alpha$ fluxes for extinction using measured values where possible, otherwise applying the average $E(B-V)=0.259$ mag, before calculating the final $\mathrm{H} \alpha$ luminosities adopting a distance of 5.45 Mpc (Herrmann et al. 2008). 
Table 4. WR features in NGC 5068. Observed flux $\left(F_{\lambda}\right)$ and extinction corrected luminosities $\left(L_{\lambda}\right)$ based on a distance of $5.45 \mathrm{Mpc}$. Values have been corrected for slit loss. Values in parentheses indicate a less secure detection $(<3 \sigma)$. Number of WR stars are based on the line luminosities for one WR star from Crowther \& Hadfield (2006), while $N(\mathrm{O} 7 \mathrm{~V})$ stars is based on $L(\mathrm{H} \alpha)$.

\begin{tabular}{|c|c|c|c|c|c|c|c|c|c|c|c|}
\hline \multirow{3}{*}{ ID } & \multirow{3}{*}{$E(B-V)$} & \multirow{3}{*}{$\begin{array}{c}F(\mathrm{~N} v-\text { III }) \\
4603-4641\end{array}$} & \multicolumn{3}{|c|}{$F_{\lambda}\left(\times 10^{-16} \mathrm{erg} \mathrm{s}^{-1} \mathrm{~cm}^{-2}\right)$} & \multicolumn{6}{|c|}{$L_{\lambda}\left(\times 10^{36} \mathrm{erg} \mathrm{s}^{-1}\right)$} \\
\hline & & & $F(\mathrm{C}$ III $)$ & $F(\mathrm{He}$ II $)$ & $F(\mathrm{He}$ II $)$ & $F(\mathrm{C}$ III $)$ & $F\left(\mathrm{C}_{\text {IV }}\right)$ & $L(\mathrm{He}$ II $)$ & $L(\mathrm{C}$ IV $)$ & WR & $N(\mathrm{WR})$ \\
\hline & & & & & & & & & & & \\
\hline 6 & 0.247 & - & - & 3.51 & 0.30 & - & - & 2.96 & - & WN4-6 & 2 \\
\hline 10 & 0.428 & - & 1.07 & - & - & - & 1.62 & - & 1.79 & WC4-5 & 1 \\
\hline 12 & 0.284 & - & 2.04 & - & - & - & 3.08 & - & 2.32 & WCE & 1 \\
\hline 13 & 0.465 & - & - & - & - & - & 1.66 & - & 2.02 & WCE(?) & 1 \\
\hline 17 & 0.230 & - & 1.05 & - & 2.04 & - & 10.2 & - & 6.67 & WC4-5 & 2 \\
\hline 19 & 0.259 & - & 4.70 & - & - & - & 5.05 & - & 3.56 & WC & 1 \\
\hline 21 & 0.595 & - & - & 1.36 & - & - & - & 3.88 & - & WNE(?) & 2 \\
\hline 35 & 0.259 & - & 11.1 & - & - & - & 8.53 & - & 6.01 & WCE & 2 \\
\hline 39 & 0.259 & - & - & 0.59 & - & - & - & 0.52 & - & $\mathrm{WN}$ & 1 \\
\hline 43 & 0.259 & - & - & 5.00 & 1.59 & - & 0.76 & 4.40 & 5.36 & WN5b & 3 \\
\hline 59 & 0.259 & - & 3.74 & - & - & - & - & - & - & WCpec? & $?$ \\
\hline 65 & 0.259 & - & 14.9 & - & - & 0.70 & 9.29 & - & 6.55 & WC6 & 2 \\
\hline 78 & 0.259 & - & - & 1.88 & - & - & - & 1.65 & - & WN6 & 1 \\
\hline 84 & 0.259 & - & 12.8 & - & - & - & 5.98 & - & 4.22 & WC4-5 & 1 \\
\hline 90 & 0.259 & - & 10.9 & - & - & - & 8.86 & - & 6.25 & WC4-5 & 2 \\
\hline 93 & 0.170 & - & - & 3.39 & - & - & - & 2.18 & - & WNE & 1 \\
\hline 94 & 0.259 & - & 8.67 & - & - & 1.23 & 3.48 & - & 2.46 & WC7 & 1 \\
\hline 99 & 0.259 & 0.80 & - & 5.36 & 0.99 & - & - & 4.62 & - & WN6 & 3 \\
\hline 104 & 0.147 & - & - & 3.86 & - & - & - & 2.29 & - & WN(?) & 1 \\
\hline 106 & 0.087 & 0.56 & - & 2.54 & - & - & - & 1.22 & - & WN7 & 1 \\
\hline 111 & 0.259 & - & - & 1.77 & - & - & - & 1.56 & - & WNL & 1 \\
\hline 112 & 0.102 & - & 1.62 & - & - & - & 2.60 & - & 1.21 & WC & 1 \\
\hline 125 & 0.259 & - & 12.1 & 1.53 & - & - & 5.85 & 1.34 & 4.12 & WC & 1 \\
\hline 128 & 0.259 & - & 4.59 & - & - & - & 3.04 & - & 2.14 & WC4-5 & 1 \\
\hline 131 & 0.180 & - & 2.35 & 0.55 & - & - & 1.12 & 0.36 & 0.64 & WC4 & 1 \\
\hline 133 & 0.259 & - & 5.67 & - & - & - & 2.73 & - & 1.93 & WC4 & 1 \\
\hline 134 & 0.181 & - & 8.90 & - & - & - & 19.3 & - & 11.1 & WC4 & 3 \\
\hline 145 & 0.259 & - & 0.85 & - & - & 0.69 & 4.12 & - & 2.90 & WC6 & 1 \\
\hline 149 & 0.125 & - & - & 2.71 & - & - & 1.51 & 1.49 & 0.75 & WNE & 1 \\
\hline 150 & 0.259 & - & 9.81 & - & - & - & 5.94 & - & 4.19 & WC4-5 & 1 \\
\hline 151 & 0.259 & - & - & 1.63 & 0.24 & - & 0.35 & 1.43 & 0.25 & WNE & 1 \\
\hline
\end{tabular}

\subsection{Star formation rate}

The SFR of the galaxy was found by placing a circular aperture with a diameter of 6.5 arcmin over the entire galaxy in our VLT/FORS1 field of view using the STARLINK package GAIA. This observed $\mathrm{H} \alpha$ flux was corrected for $[\mathrm{N}$ II] emission $([\mathrm{N} I] / \mathrm{H} \alpha=0.155)$ and extinction $(E(B-V)=0.259 \mathrm{mag})$ producing a total $\mathrm{H} \alpha$ luminosity of $5.68 \times 10^{40} \mathrm{erg} \mathrm{s}^{-1}$. Following the relations of Kennicutt (1998) the corrected $\mathrm{H} \alpha$ flux luminosity was used to calculate the number of ionizing photons, from which the SFR of the galaxy is inferred. The VLT/FORS1 images of NGC 5068 suggest a SFR $=0.45 \mathrm{M}_{\odot} \mathrm{yr}^{-1}$, excluding the $\mathrm{H} \alpha$ emission which lies in the $\mathrm{CCD}$ chip gap and also extended emission beyond our field of view.

Table 5 shows a comparison of our analysis with previous work. The result from Ryder \& Dopita (1994) is substantially higher than the other estimates, but unfortunately no observed flux measurements are available so no further comparison with this result can be made. In addition, the observations of Hodge (1974) are consistent with our estimate, however, they only observe the inner part of the galaxy and again no measured fluxes are available for comparison. We include both of these results in Table 5 only for completeness. The flux measurements of Kennicutt et al. (2008) are the most complete since the $13 \operatorname{arcmin}^{2}$ field of view, which is double our own, contains all the $\mathrm{H} \alpha$ emission from the galaxy. The SFR they derive is $0.53 \mathrm{M}_{\odot} \mathrm{yr}^{-1}$, however, they only correct for foreground extinction, and find a larger $[\mathrm{N} \mathrm{II}] / \mathrm{H} \alpha$ contribution of 0.209 which was estimated using empirical scaling relation since no direct observations were available.

The $\mathrm{H} \alpha$ flux from the archival Cerro Tololo Inter-American Observatory (CTIO) images of NGC 5068 used by Kennicutt et al. (2008) exceeds our measurements by $\sim 30$ per cent, which is unsurprising considering the smaller field of view and detector gaps in our VLT images. Therefore, we adopt the observed flux from Kennicutt et al. (2008) of $F(\mathrm{H} \alpha+[\mathrm{N}$ II] $)=1.48 \times$ $10^{-11} \mathrm{erg} \mathrm{s}^{-1} \mathrm{~cm}^{-2}$. Since $[\mathrm{N} \mathrm{II}] / \mathrm{H} \alpha$ decreases with metallicity from the metal-rich inner disc to the metal-poor outer disc, we correct for three separate values of $[\mathrm{N} I I] / \mathrm{H} \alpha$ at $r / R_{25}=0-0.28$, $0.29-0.69$ and $0.70-1$. Using the measured values of $[\mathrm{N} I] / \mathrm{H} \alpha$ from sources listed in Table 3 we find the average $[\mathrm{N} \Pi] / \mathrm{H} \alpha$ value within each $r / R_{25}$ range, resulting in $[\mathrm{N} I] / \mathrm{H} \alpha=0.28,0.17$ and 0.08 , from the inner to outer disc, respectively. We find a $F(\mathrm{H} \alpha)=1.22 \times 10^{-11} \mathrm{erg} \mathrm{s}^{-1} \mathrm{~cm}^{-2}$ and calculate total an $\mathrm{H} \alpha$ luminosity of $7.93_{-1.65}^{+1.32} \times 10^{40} \mathrm{erg} \mathrm{s}^{-1}$ for NGC 5068, after correction for the $[\mathrm{N}$ II] emission and average extinction from our GMOS spectroscopy. This corresponds to a global SFR $=0.63_{-0.13}^{+0.11} \mathrm{M}_{\odot} \mathrm{yr}^{-1}$ adopting the Kennicutt (1998) calibration. The errors on 

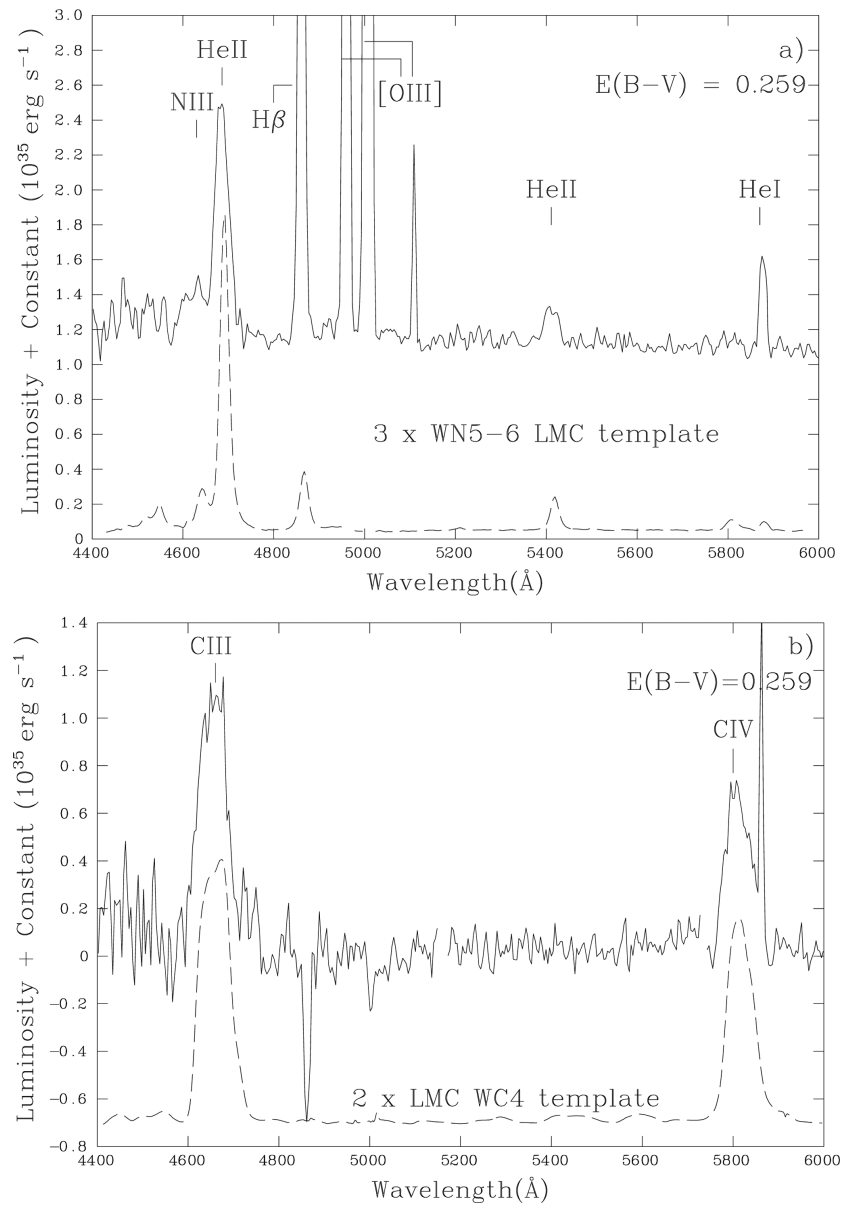

Figure 8. Dereddened spectra of (a) WN (source \#99) and (b) WC (source \#90) stars in NGC 5068 (solid lines), corrected for a distance of $5.45 \mathrm{Mpc}$, with LMC WR template spectra (dashed lines) offset for comparison. The WR emission lines (and nebular lines in a) are indicated and both sources have been extinction corrected by $E(B-V)=0.259$ mag. The oversubtracted $\mathrm{H} \beta$ line in (b) is likely due to a bad sky subtraction.
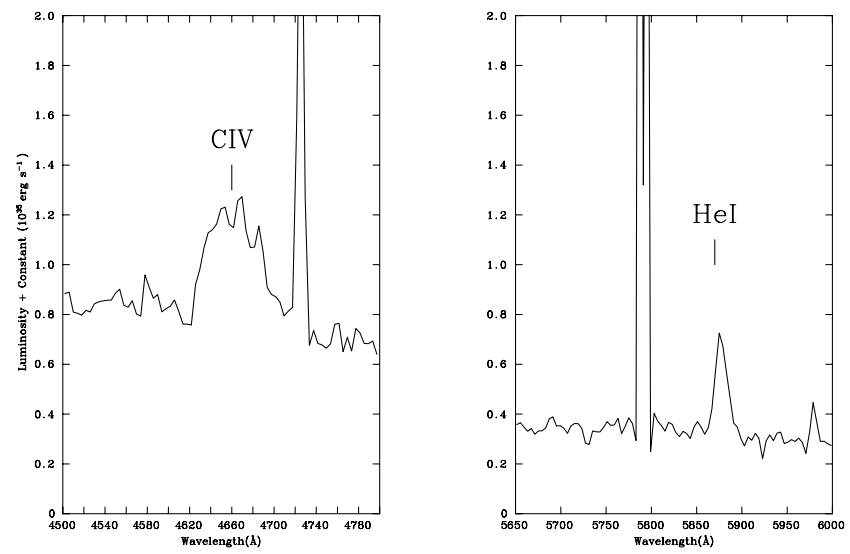

Figure 9. Flux-calibrated spectra of source \#59 showing the presence of broad $\lambda 4660$ emission suggestive of C IV $\lambda 4660$ (left), albeit without neither $\mathrm{C}_{\text {IV }} \lambda 5808$ nor $\mathrm{C}$ III $\lambda 5696$ emission. The line at $\sim 5780 \AA$ is an unsubtracted sky line. the SFR are derived from the errors on $E(B-V)$ and on the distance of NGC 5068 from Herrmann et al. (2008).

\subsection{O star population}

The $\mathrm{H} \alpha$ luminosity of $7.93_{-1.65}^{+1.32} \times 10^{40} \mathrm{erg} \mathrm{s}^{-1}$ equates to $\sim 6000_{-1500}^{+2000}$ O7V stars, assuming an ionizing flux $Q_{0}=10^{49}$ photons s $^{-1}$ for a typical O7V star in a low-metallicity environment (Vacca 1994). However, in reality not all of the $\mathrm{O}$ stars will be $\mathrm{O} 7$ dwarfs but will span the entire spectral range. Starburst models by Schaerer \& Vacca (1998) can be used to estimate the true number of O stars for a given age, $t$, using the parameter $\eta_{0}(t)$. Unfortunately the spiral arms of NGC 5068 undergo continuous star formation and hence the age could range from 3 to $10 \mathrm{Myr}$, the lifetime of O stars. Consequently we follow Bibby \& Crowther (2010) who used the 30 Doradus region of the LMC, and the Small Magellanic Cloud (SMC), as proxy to determine a uniform correction factor of $N(\mathrm{O}) / N(\mathrm{O} 7 \mathrm{~V}) \sim$ 1.5 , suggesting a total $\mathrm{O}$ star population of $\sim 9000_{-2000}^{+1500}$.

Hodge (1974) identified $83 \mathrm{H}$ II regions in NGC 5068, including some giant $\mathrm{H}$ II regions (GHR), formally defined as regions in which $Q_{0}$ exceeds $10^{50}$ photons s $^{-1}$ (Conti, Crowther \& Leitherer 2008). A catalogue of the 29 brightest $\mathrm{H}$ II regions in NGC 5068 is presented in Table 6. The observed $\mathrm{H} \alpha$ flux within a given diameter, $d_{\mathrm{ap}}$, has been continuum subtracted, corrected for the contribution of [N II], plus an average NGC 5068 extinction unless this has been individually measured (see Section 5). From the list of $\mathrm{H}$ II regions in Table $6, \sim 17$ qualify as GHR, albeit these are dependent upon extinction and distance uncertainties. Note that NGC 5068 hosts three very bright $\mathrm{H}_{\text {II }}$ regions, which each host over 100 equivalent $\mathrm{O} 7$ dwarfs, reminiscent of the brightest $\mathrm{H}$ II regions in the Milky Way (e.g. NGC 3603), albeit somewhat more modest in ionizing output than the 30 Doradus region of the LMC (Kennicutt 1984).

Mindful that Table 6 may be incomplete due to the detector gap in the FORS 1 images, we inspected the archival $\mathrm{H} \alpha$ CTIO images of Kennicutt et al. (2008) but found that no substantial $\mathrm{H}$ II regions were present in this region.

Table 6 provides cross-matches to the catalogue of Hodge (1974), and also indicates which bright $\mathrm{H}_{\mathrm{II}}$ regions host WR candidates. 20 per cent of the WR candidates lie within the most prominent $\mathrm{H}$ II regions, although considering the entire 160 candidate list, 50 per cent lie within bright (compact) $\mathrm{H}_{\text {II }}$ regions, 25 per cent are associated with faint (diffuse) nebulosity and 25 per cent are not associated with any $\mathrm{H}_{\text {II }}$ region (Table 2).

\section{THE GLOBAL WR POPULATION OF NGC 5068}

The spectroscopic observations of $30 \mathrm{WR}$ candidates identify 18 WN stars and 24 WC stars in NGC 5068. We use the photometric properties of these confirmed WR stars to infer the likelihood that the remaining WR candidates are indeed true WR stars, and in some cases assess their WR subtype. In addition we take account of the detection limits and photometric completeness of our survey to derive the global WR population of NGC 5068.

\subsection{Nature of the remaining candidates}

In Section 2.3 we established that our photometric and spectroscopic $\lambda 4684$ excesses were in good agreement (recall Fig. 5). Therefore, we can assess the nature of the remaining candidates based upon the properties of our confirmed WR sources. Fig. 10 compares the 
Table 5. Integrated $\mathrm{H} \alpha$ flux/luminosity for NGC 5068, with respect to Kennicutt et al. (2008) (K08) and other previous studies. Fluxes are in units of $10^{-11} \mathrm{erg} \mathrm{s}^{-1} \mathrm{~cm}^{-2}$, and luminosities are in units of $10^{40} \mathrm{erg} \mathrm{s}^{-1}$. The inner, middle and outer regions correspond to $r / R_{25}=0-0.28,0.29-0.69$ and $0.7-1.0$, respectively.

\begin{tabular}{lcccccccc}
\hline Work & $F\left(\mathrm{H} \alpha+\left[\mathrm{N}_{\mathrm{II}}\right]\right)$ & {$[\mathrm{N} \mathrm{II}] / \mathrm{H} \alpha$} & $E(B-V)$ & $\begin{array}{c}D \\
(\mathrm{Mpc})\end{array}$ & $\begin{array}{c}L(\mathrm{H} \alpha) \\
\log \left(Q_{0}\right) \\
\left(\mathrm{s}^{-1}\right)\end{array}$ & $N(\mathrm{O} 7 \mathrm{~V})$ & $\begin{array}{c}\mathrm{SFR} \\
\left(\mathrm{M}_{\odot} \mathrm{yr}^{-1}\right)\end{array}$ \\
\hline This work & 1.04 & 0.155 & 0.259 & 5.45 & 5.68 & 52.62 & 4200 & 0.45 \\
K08 & 1.48 & 0.209 & 0.090 & 6.20 & 6.61 & 52.69 & 4900 & 0.53 \\
Inner & 0.23 & 0.28 & 0.259 & 5.45 & 1.07 & 51.90 & 800 & 0.09 \\
Middle & 1.04 & 0.18 & 0.259 & 5.45 & 5.53 & 52.62 & 4200 & 0.45 \\
Outer & 0.21 & 0.08 & 0.259 & 5.45 & 1.26 & 51.97 & 900 & 0.10 \\
Total & 1.48 & & & & 7.93 & 52.77 & 5900 & 0.63 \\
Ryder \& Dopita (1994) & & & & & & & 3.0 \\
Hodge (1974) & & & & & & & 0.35 \\
\hline
\end{tabular}

Table 6. The flux, luminosity and $N(\mathrm{O})$ stars for the brightest $29 \mathrm{H}$ II regions in NGC 5068. Continuum subtracted fluxes, $F(\mathrm{H} \alpha)$, are in units of $10^{-14} \mathrm{erg} \mathrm{s}^{-1} \mathrm{~cm}^{-2}$ and are corrected for $\left[\mathrm{N}_{\mathrm{II}}\right]$ emission using $\mathrm{H} \alpha /\left[\mathrm{N}_{\mathrm{II}}\right]=0.155$. Luminosities are in units of $10^{38} \mathrm{erg} \mathrm{s}^{-1}$, assuming a distance of $5.45 \mathrm{Mpc}$. The diameter of the aperture, $d_{\mathrm{ap}}$, is given in arcsec. Also noted is whether WR stars have been identified within these $\mathrm{H}$ II regions.

\begin{tabular}{|c|c|c|c|c|c|c|c|c|c|c|}
\hline ID & RA & Dec. & $d_{\mathrm{ap}}$ & $F(\mathrm{H} \alpha)$ & $E(B-V)$ & $L(\mathrm{H} \alpha)$ & $\log \left(Q_{0}\right)$ & $N(\mathrm{O} 7 \mathrm{~V})$ & Hodge & WR \\
\hline Н II \#1 & $13: 18: 42.8$ & $-21: 01: 24$ & 5 & 19.1 & 0.259 & 12.3 & 50.96 & 91 & H82 & - \\
\hline Н II \#2 & $13: 18: 44.5$ & $-21: 01: 14$ & 5 & 1.72 & 0.259 & 1.11 & 49.92 & 8 & H79 & - \\
\hline Н II \#3 & $13: 18: 44.8$ & $-21: 00: 51$ & 4 & 1.48 & 0.259 & 0.96 & 49.85 & 7 & H77 & 11 \\
\hline Н II \#4 & $13: 18: 45.8$ & $-21: 02: 31$ & 5 & 4.28 & 0.259 & 2.77 & 50.31 & 20 & $\mathrm{H} 73$ & 14,16 \\
\hline Н II \#5 & $13: 18: 47.0$ & $-21: 00: 50$ & 5 & 1.69 & 0.259 & 1.09 & 49.91 & 8 & H86 & - \\
\hline Н II \#6 & $13: 18: 47.1$ & $-21: 00: 34$ & 3 & 0.76 & 0.595 & 1.07 & 49.90 & 9 & & 21 \\
\hline Н II \#7 & $13: 18: 47.3$ & $-21: 03: 53$ & 6 & 3.18 & 0.259 & 2.05 & 50.18 & 15 & & 23,26 \\
\hline $\mathrm{H}_{\text {II }} \# 8$ & $13: 18: 48.0$ & $-21: 03: 11$ & 4 & 1.63 & 0.259 & 1.05 & 49.89 & 8 & H65 & - \\
\hline Н II \#9 & $13: 18: 48.1$ & $-21: 00: 49$ & 7 & 16.9 & 0.259 & 10.9 & 50.91 & 81 & H60 & 34,36 \\
\hline Н II \#10 & $13: 18: 48.5$ & $-21: 00: 17$ & 4 & 1.75 & 0.259 & 1.13 & 49.92 & 8 & H59 & 40 \\
\hline Н & $13: 18: 49.0$ & $-21: 00: 22$ & 4 & 4.08 & 0.259 & 2.64 & 50.29 & 20 & H54 & 41 \\
\hline $\mathrm{H}_{\text {II }} \# 12$ & $13: 18: 49.3$ & $-21: 00: 23$ & 4 & 4.98 & 0.259 & 3.22 & 50.38 & 24 & $\mathrm{H} 50$ & 45 \\
\hline $\mathrm{H}_{\text {II }} \# 13$ & $13: 18: 49.8$ & $-21: 02: 29$ & 5 & 4.28 & 0.259 & 2.77 & 50.31 & 20 & H48 & $52,53,56$ \\
\hline $\mathrm{H}_{\text {II }} \# 14$ & $13: 18: 49.9$ & $-21: 03: 23$ & 10 & 28.2 & 0.259 & 18.3 & 51.13 & 135 & H49 & $49,55,58,60$ \\
\hline $\mathrm{H}_{\text {II }} \# 15$ & 13:18:50.7 & $-21: 02: 06$ & 5 & 1.39 & 0.259 & 0.90 & 49.82 & 7 & $\mathrm{H} 42$ & 69,70 \\
\hline Н & $13: 18: 51.3$ & $-21: 04: 09$ & 5 & 3.22 & 0.259 & 2.08 & 50.19 & 15 & $\mathrm{H} 40$ & $73,75,76$ \\
\hline Н нІ \#17 & $13: 18: 51.7$ & $-21: 01: 18$ & 5 & 7.16 & 0.259 & 4.63 & 50.53 & 34 & 78 & \\
\hline $\mathrm{H}_{\text {II }} \# 18$ & $13: 18: 51.8$ & $-21: 00: 50$ & 4 & 1.86 & 0.259 & 1.20 & 49.95 & 9 & H36 & 81 \\
\hline Н II \#20 & 13:18:53.2 & $-21: 02: 45$ & 4 & 2.72 & 0.259 & 1.76 & 50.12 & 13 & H33 & 103 \\
\hline $\mathrm{H}_{\text {II }} \# 21$ & $13: 18: 54.7$ & $-21: 03: 10$ & 7 & 4.34 & 0.259 & 2.81 & 50.32 & 21 & $\mathrm{H} 29$ & 116 \\
\hline Н & $13: 18: 54.9$ & $-21: 04: 04$ & 4 & 2.94 & 0.259 & 1.32 & 49.99 & 10 & & 121 \\
\hline $\mathrm{H}_{\text {II }} \# 23$ & $13: 18: 55.2$ & $-21: 02: 42$ & 4 & 1.95 & 0.259 & 1.26 & 49.97 & 9 & & - \\
\hline $\mathrm{H}_{\text {II }} \# 24$ & $13: 18: 56.7$ & $-21: 00: 33$ & 4 & 1.53 & 0.259 & 9.87 & 49.86 & 7 & H14 & - \\
\hline Н & $13: 18: 56.8$ & $-21: 04: 13$ & 4 & 5.18 & 0.181 & 2.79 & 50.32 & 21 & & 134 \\
\hline $\mathrm{H}_{\text {II }} \# 27$ & $13: 18: 57.3$ & $-21: 00: 47$ & 15 & 29.1 & 0.259 & 18.8 & 51.14 & 139 & H13 & 139 \\
\hline Н & $13: 18: 58.2$ & $-21: 02: 28$ & 4 & 2.72 & 0.259 & 1.76 & 50.11 & 13 & & 145 \\
\hline $\mathrm{H}_{\text {II }} \# 30$ & $13: 18: 59.2$ & $-21: 00: 06$ & 4 & 1.20 & 0.259 & 0.78 & 49.76 & 6 & $\mathrm{H} 10$ & - \\
\hline $\mathrm{H}_{\text {II }} \# 31$ & 13:19:00.8 & $-21: 02: 47$ & 9 & 3.21 & 0.259 & 20.8 & 51.19 & 154 & $\mathrm{H} 7$ & 152 \\
\hline $\mathrm{H}_{\text {II }} \# 32$ & 13:19:03.1 & $-21: 01: 00$ & 4 & 1.51 & 0.259 & 0.98 & 49.86 & 7 & $\mathrm{H} 2$ & - \\
\hline
\end{tabular}

photometric $m_{4684}-m_{4781}$ excess against the absolute $\lambda 4684$ magnitude of the WR candidates identified in the VLT/FORS1 imaging. Those candidates that have a spectroscopically determined subtype are plotted accordingly, while those candidates for which spectroscopy was not obtained are plotted by the open triangles and the non-WR sources are marked with an ' $\mathrm{x}$ '.

In addition to the sources plotted in Fig. 10 there are three confirmed WC, two confirmed WN sources and 25 additional non-WR sources which do not possess photometry in one or both filters. Photometry for such sources is not available if the source is extended, in a crowded region, or too faint in the $\lambda 4781$ observation.
As a result of their strong emission lines, WC stars have significantly larger photometric $\lambda 4684$ excesses relative to $\mathrm{WN}$ stars (Conti \& Massey 1989). Our spectroscopic observations reflect this, since all sources with $\lambda 4686$ excesses of at least -1.5 mag are spectroscopically confirmed as WC stars, as indicated in Fig. 10. Consequently, we can fairly reliably infer that the remaining five WR candidate regions with equally large $\lambda 4684$ excesses also host WC stars.

In order to estimate the number of WR stars for each photometric candidate, we convert $m_{4684}$ and $m_{4781}$ magnitudes into fluxes, and estimate emission line fluxes by subtracting $f_{4781}$ from 


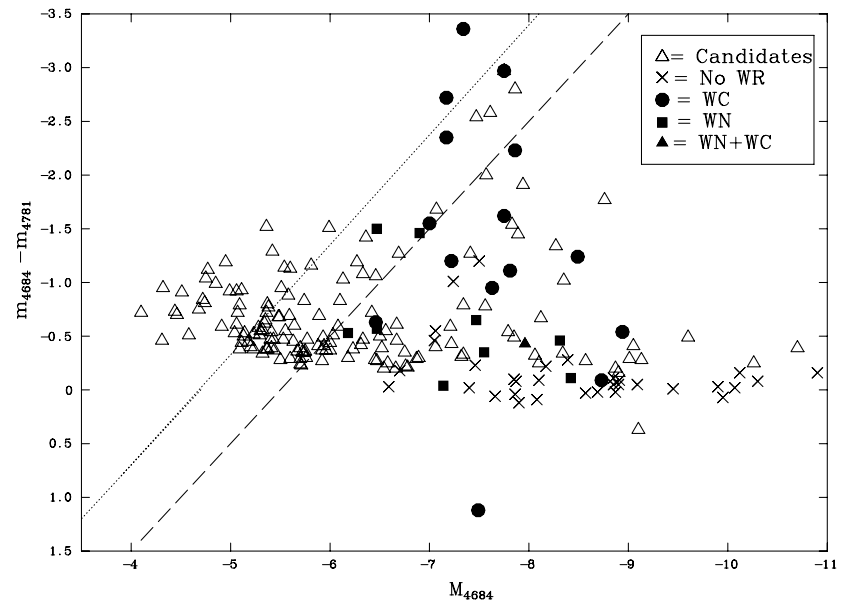

Figure 10. Absolute He iा $\lambda 4686$ magnitude versus He iा $\lambda 4686$ excess for WR candidates in NGC 5068. Different symbols are used for WR subtypes confirmed by spectroscopy and are marked as shown in the legend. The 100 and 50 per cent completeness limits are indicated by the dashed and dotted lines, respectively.

$f_{4684}$, and then follow the same approach as for the spectroscopic data sets. We have compared photometric and spectroscopic $4603 / 4650 / 4686$ fluxes for 24 sources in common, and find spectroscopic fluxes are typically $\sim 50$ per cent higher. In part, this arises from the relatively narrow full width at half-maximum (FWHM) of the $\lambda 4684$ bandpass with respect to broad WR emission (e.g. FWHM $\sim 55 \AA$ for \#112). Therefore, photometric flux estimates should provide useful lower limits to the WR population of each source.

For the spectroscopically observed candidates with photometric excesses between -0.6 and $-1.5 \mathrm{mag}$, all are confirmed except in one instance, so these too are likely to host WR stars. It is less straightforward to assign a WN or WC subtype to these candidates since they overlap in $\lambda 4684$ excesses since the stellar continua from other stars dilute the WR emission lines, resulting in smaller excesses; this is discussed further in Section 6.2. Still, since WN subtypes possess intrinsically weak emission, they are more likely to correspond to the faintest sources with modest excesses.

Therefore, we assign a photometric WN subtype for sources fainter than $M_{4686} \sim-7.5$, and photometric WC subtypes otherwise. For the spectroscopic sources with weaker excesses below -0.6 mag, only a small percentage ( $\sim 20$ per cent $)$ were confirmed as genuine WR stars. Therefore, for such stars to be considered as a photometric WR star we require a photometric $m_{4684}-m_{4781}$ excess of at least $3 \sigma$, plus an excess in $\lambda 4686$ with respect to the $V$ band. Such stars are most likely to be WN subtypes, except for the very brightest sources with $M_{4686} \sim-8.5 \mathrm{mag}$.

In total, we estimate an additional $43 \mathrm{WN}$ stars to the $18 \mathrm{spec}-$ troscopically identified, plus an additional $11 \mathrm{WC}$ stars to the 24 obtained from spectroscopy, i.e. $61 \mathrm{WN}$ and $35 \mathrm{WC}$ stars in total.

In addition to photometric sources, 17 candidates without photometric information have yet to be incorporated. From the seven such cases spectroscopically observed, five were confirmed as WR stars, so we shall assume that a similar fraction (12 from 17) of the candidates are genuine WR stars. However, we do not attempt to discriminate between $\mathrm{WN}$ and WC subtypes. Our estimate for the grand total of WR stars in NGC 5068 is therefore $\sim 110$, with $N(\mathrm{WN}) / N(\mathrm{WC}) \geq 1.7$.

\subsection{Completeness}

From our 160 candidate WR sources in NGC 5068 we have estimated a global total of $\sim 110 \mathrm{WR}$ stars. However, in order to assess the true WR population we must assess how many WR stars were not detected due to the limiting magnitudes our images.

The detection limits plotted as the dashed (100 per cent) and solid (50 per cent) lines in Fig. 10 represent the depth of our FORS1 imaging based on the magnitude of all the objects detected in the image (including non-WR sources). The $\lambda 4684$ magnitude range of objects was 16-27 mag with the turnover of the distribution at $\sim 24.0 \mathrm{mag}$ corresponding to a 100 per cent detection limit of $M_{4684}=-5.4 \mathrm{mag}$ for an average extinction of $E(B-V)=0.259$ mag and distance of $5.45 \mathrm{Mpc}$.

The main uncertainty on the assessing completeness of a global WR population is identifying unresolved regions hosting WR stars. While relatively isolated and resolved WR sources are easily identified from narrow-band imaging, unresolved regions hosting multiple early-type stars can dilute the WR emission and hide the WR source completely. How many candidates we did not detect due to dilution?

Bibby \& Crowther (2010) derived the total completeness of their WR study of NGC 7793 from comparison with the complete WR population of the LMC by tracing how the $\lambda 4684$ emission from a representative sample of LMC WR stars would be diluted if the spatial resolution of the LMC images were the same as their NGC 7793 imaging $(1.3 \operatorname{arcsec} \sim 20 \mathrm{pc})$. By applying the detection limits of the narrow-band imaging to the diluted LMC sample they estimated the percentage of the known LMC WR stars that would not have been detected.

The larger distance of $5.45 \mathrm{Mpc}$ to NGC 5068 together with an improved spatial resolution of 0.8 arcsec combine to produce a similar physical scale of $\sim 20 \mathrm{pc}$. This is presented in Fig. 11, from which we estimate that $\sim 85$ per cent of the WR stars in the LMC would be detected in our survey, based on the detection limits and spatial resolution of our narrow-band images, indicating a global WR population of $\sim 130$.

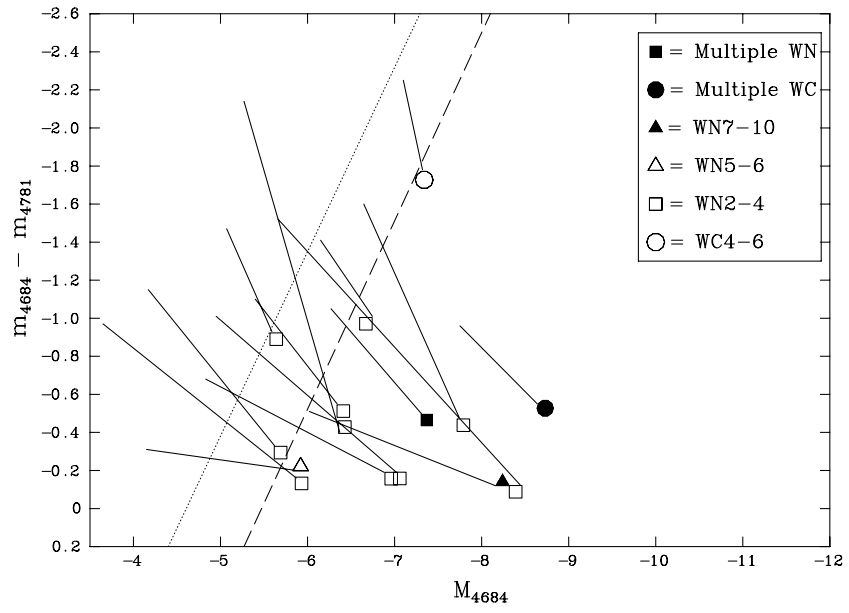

Figure 11. Dilution of the $\lambda 4686$ emission line for a representative sample of WR stars in the LMC, adapted from Bibby \& Crowther (2010). We have applied our 100 and 50 per cent detection limits from our VLT/FORS narrow-band imaging, indicated by the dashed and dotted lines, respectively, to assess the completeness of our WR survey of NGC 5068. 14 out of 15 LMC WR stars would have been detected indicating that our survey should be $\sim 90$ per cent complete. 
Overall, since WC stars have a stronger excess relative to WN stars it is likely that we are close to $\sim 100$ per cent complete in WC stars in NGC 5068. Early-type WN (WNE) stars have larger excesses than late-type WN (WNL) stars, but are significantly fainter visually, so we expect to be missing a significant percentage of WNE stars, but also be relatively complete in WNL stars. However, the diluted photometric excesses of four out of the 15 representative WR stars were $<0.3 \mathrm{mag}$ and fainter than $M_{4686}=-7 \mathrm{mag}$ (Fig. 11). Some such faint, weak excess sources - primarily WNE stars - will have been included in our catalogue, but would most likely be listed under 'no WR' since they are not photometrically statistically significant. Indeed we see several examples of such sources in our survey such as source \#28, which is a genuine WNE star with an excess of only $0.18 \mathrm{mag}$ and $M_{4684}=-6.53 \mathrm{mag}$. Our survey of WNE stars is almost certainly incomplete from the perspective that the large excesses, which enable us to identify these faint stars, are diluted leaving only a faint excess which is hard to detect from ground-based images. Therefore, we predict a global WR population of $\sim 170$ for NGC 5068 due to incompleteness for the faintest 25 per cent of WR stars.

\subsection{Predicted WR population}

In Section 5.2 we used the SFR of the galaxy to determine that there were $\sim 9000_{-2000}^{+1000} \mathrm{O}$ stars in NGC 5068. We can use this information to infer the expected number of WR stars based on empirical values and independently assess the completeness of our survey. The ratio of WR to O stars varies with metallicity, as a result of the metallicity dependence of winds from massive stars, ranging from $\sim 0.03$ in the LMC to $\sim 0.15$ in the solar neighbourhood (Crowther 2007). The average metallicity we find for NGC 5068 is $\log (\mathrm{O} / \mathrm{H})+12=8.44 \pm$ 0.17 (recall Table 3 ), similar to the LMC, so assuming a similar WR to O ratio for NGC 5068 we would anticipate a WR population of $\sim 270_{-60}^{+45}$ based upon its $\mathrm{O}$ star population of $\sim 9000_{-2000}^{+1500}$.

If the WR population of NGC 5068 is $\sim 270$, our earlier estimate is 40 per cent incomplete. One possible explanation is that the percentage of weak-lined, faint WN stars could be considerably higher relative to other WR subtypes, as indeed is the case in the LMC. Ideally, deep, high-resolution (space-based) imaging of NGC 5068 would be required, together with further follow-up spectroscopy to confirm this.

\section{COMPARISON TO NGC 7793}

WR surveys beyond the Local Group have been undertaken on several nearby, spiral galaxies (e.g. Crowther \& Hadfield 2006; Hadfield \& Crowther 2007; Bibby \& Crowther 2010). The survey of NGC 7793 by Bibby \& Crowther (2010) is an ideal galaxy with which to compare NGC 5068 given their similar physical properties.

NGC 7793 is a Sculptor group SA(s)d galaxy which is slightly nearer to us at 3.91 Mpc (Karachentsev 2003) than NGC 5068. Basic properties of each galaxy are presented in Table 7 which shows that although the galaxies have similar physical sizes and central metallicities, the abundance gradient of NGC 5068 is nearly twice as steep as NGC 7793. We note that both studies use the N2 and O3N2 calibrations so are subject to the same systematic errors hence a comparison of $\mathrm{H}$ II region abundances is not biased.

Also included in Table 7 is a comparison of their SFRs, with NGC 506850 per cent higher than NGC 7793. A similar number of WC stars have been spectroscopically confirmed in both galaxies, and although a smaller fraction of WN have been observed
Table 7. Global properties of NGC 5068 and NGC 7793, along with a census of their massive stellar populations. Properties of NGC 7793 are taken from Bibby \& Crowther (2010) unless otherwise stated. The number of WR candidates and the percentage that are spectroscopically confirmed are listed along with the confirmed number of WN and WC stars.

\begin{tabular}{|c|c|c|}
\hline Name & NGC 7793 & NGC 5068 \\
\hline Hubble type & $\mathrm{SA}(\mathrm{s}) \mathrm{d}$ & $\mathrm{SB}(\mathrm{d})$ \\
\hline Distance (Mpc) & $3.91^{a}$ & $5.45^{b}$ \\
\hline$R_{25}(\operatorname{arcmin})$ & $4.65^{c}$ & $3.62^{c}$ \\
\hline$R_{25}(\mathrm{kpc})$ & $5.3^{c}$ & $5.7^{c}$ \\
\hline $\operatorname{SFR}\left(\mathrm{M}_{\odot} \mathrm{yr}^{-1}\right)$ & 0.45 & 0.63 \\
\hline $\log (\mathrm{O} / \mathrm{H})+12_{\text {central }}$ & 8.61 & 8.74 \\
\hline Gradient $\left(\operatorname{dex~} \mathrm{kpc}^{-1}\right)$ & -0.07 & -0.11 \\
\hline $\log (\mathrm{O} / \mathrm{H}))+12_{\text {mean }}$ & 8.40 & 8.44 \\
\hline$N(\mathrm{O} 7 \mathrm{~V})$ & 4200 & 6000 \\
\hline$N(\mathrm{O})$ & 6250 & 9000 \\
\hline WR candidates & 74 & 160 \\
\hline Spectra obtained & 53 per cent & 42 per cent \\
\hline$N(\mathrm{WN})_{\text {spect }}$ & 27 & 18 \\
\hline$N(\mathrm{WC})_{\text {spect }}$ & 25 & 24 \\
\hline$N(\mathrm{WR})_{\text {total }}$ & 105 & 170 \\
\hline$N(\mathrm{WR}) / N(\mathrm{O})$ & 0.017 & 0.019 \\
\hline
\end{tabular}

in NGC 5068 its WR population is anticipated to be $\sim 40$ per cent higher.

\subsection{Comparison with evolutionary models}

Evolutionary models of massive stellar populations (Meynet \& Maeder 2005; Eldridge \& Vink 2006) predict a correlation in the WR/O star and WC/WN star ratios as a function of metallicity, which has been confirmed by observations of Local Group galaxies (Massey 1996).

Fig. 12 compares the empirical $N(\mathrm{WR}) / N(\mathrm{O})$ ratio of different galaxies at a range of metallicities with evolutionary predictions from Eldridge \& Vink (2006) and Meynet \& Maeder (2005). The single and binary models of Eldridge \& Vink (2006) which include metallicity-dependent WR winds are a somewhat better overall match to the observed data than the single star predictions from Meynet \& Maeder (2005). However, neither model is successful in tracing the $N(\mathrm{WR}) / N(\mathrm{O})$ ratio with both appearing to overestimate the number of WR stars, e.g. $N(\mathrm{WR}) / N(\mathrm{O})=0.019_{-0.003}^{+0.005}$ for NGC 5068 (similar to NGC 7793) is lower than both sets of predictions. However, as discussed above, if we have underestimated the early-type WN population, which contribute $\sim 50$ per cent of the WR stars in the LMC, then the ratio could readily be as high as $N(\mathrm{WR}) / N(\mathrm{O}) \sim 0.03$ which would be in good agreement with theoretical predictions of Eldridge \& Vink (2006).

\section{THE SPATIAL DISTRIBUTION OF WR STARS}

Different ccSN subtypes appear to be located in different regions of their host galaxies. For example, Type II SNe, whose progenitors have been identified as RSG (see Smartt 2009 for review), are found to follow the distribution of the host galaxy light whereas Type $\mathrm{Ib}$, and more so Ic SNe, are preferentially located in the brightest 


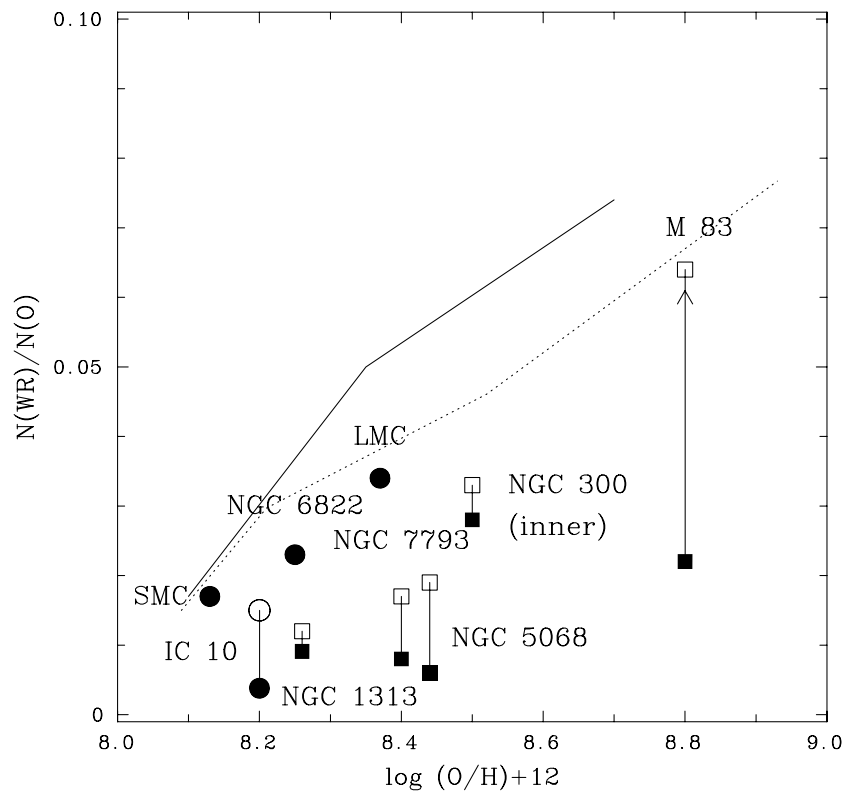

Figure 12. Comparison between observed $N(\mathrm{WR}) / N(\mathrm{O})$ ratios in nearby and Local Group galaxies with predictions from the evolutionary models of Eldridge \& Vink (2006) (dotted) and Meynet \& Maeder (2005) (solid). Filled symbols are results from spectroscopy and open symbols account for completeness.

regions (Fruchter et al. 2006; Kelly et al. 2008). Indeed, there is apparently no association between Type II SNe and $\mathrm{H}$ II regions according to Anderson \& James (2008), whereas Type Ib and especially Type Ic $\mathrm{SNe}$ are intimately associated with bright $\mathrm{H}$ II regions. In addition, recent work by Modjaz et al. (2011) has revealed that Type Ic SNe are located in more metal-rich regions than Type Ib SN. If $\mathrm{WN}$ and $\mathrm{WC}$ stars are indeed the progenitors of Type Ib and Type Ic SNe, respectively, then they should follow similar distributions.

We use a broad-band image of NGC 5068 from the Digitized Sky Survey (DSS) since the chip gap in our VLT/FORS1 images would cause the light distribution of the galaxy to be non-uniform. We subtract all the foreground stars before degrading the image, to mimic the spatial resolution of the SDSS galaxies used in Kelly et al. (2008), and remove all the background light (Leloudas et al. 2010). Although we can assess the likely WR nature of the remaining WR candidates we only consider the spatial distribution of spectroscopically confirmed WN and WC stars that have been detected above the $3 \sigma$ level.

Using an IDL routine we obtain the fractional flux at the location of each WR star as a function of the number of stars. This is presented in Fig. 13. In addition, we performed a KS test to assess the probability that two distributions were from the same parent population; the results of each comparison are presented in Table 8.

From an initial visual inspection, the distribution of the WN stars appears to be most consistent with the distribution of the Type II $\mathrm{SNe}$ in the brightest and faintest $\sim 30$ per cent of the galaxy but lie in between the Type Ib and Type Ic-bl SNe distributions in the moderately bright regions (30-70 per cent fractional flux). The results from the KS test are consistent with the qualitative analysis and show that the WN stars in NGC 5068 are most likely to be the parent population of Type Ib and Type II SNe, but does not rule out WN stars as the progenitors of Type Ic-bl SNe.

One possible explanation for the deviation of the $\mathrm{WN}$ from the Type $\mathrm{Ib} / \mathrm{c}$ distributions in brighter regions is that WNE stars with low $\lambda 4686$ excesses are extremely difficult to detect in bright re-

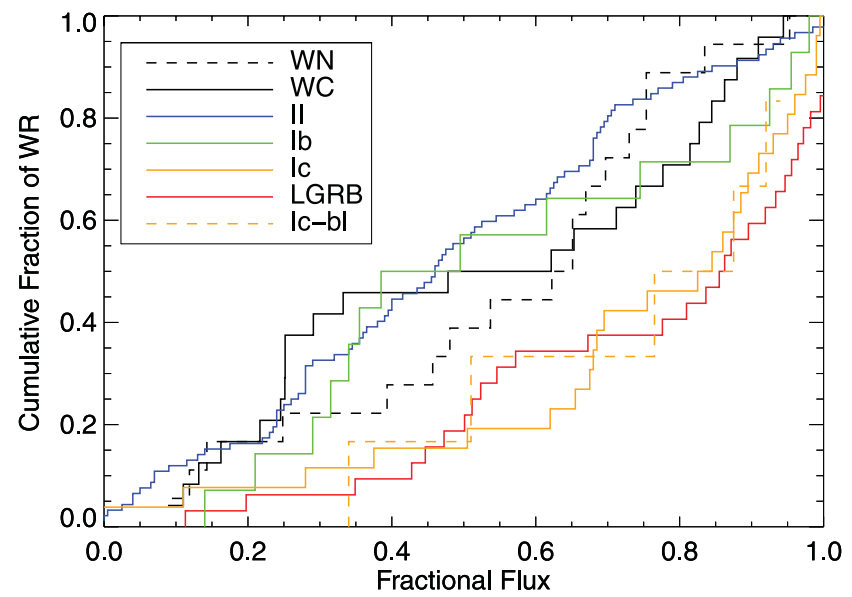

Figure 13. A comparison of the distribution of WN and WC stars in NGC 5068 (dashed and solid black lines, respectively) relative to the distribution of light in the host galaxy. Plots of supernova and gamma-ray burst (GRB) distributions are also shown, taken from Kelly et al. (2008) and Fruchter et al. (2006), respectively.

Table 8. Results of the KS test performed on the spatial distribution of WR stars in NGC 5068 relative to the galaxy light distribution. This reveals that WN stars are most likely the progenitors of Type II or Type Ib SNe, while WC stars are more likely to produce a broad-lined Type Ic $\mathrm{SNe}$.

\begin{tabular}{ccccccc}
\hline & WC & Type II & Type Ib & Type Ic & Type Ic-bl & LGRB \\
\hline WN & 0.460 & 0.417 & 0.512 & 0.033 & 0.361 & 0.004 \\
WC & - & 0.368 & 0.585 & 0.070 & 0.444 & 0.044 \\
\hline
\end{tabular}

gions at low spatial resolution as discussed in Section 6.2. A deep, high spatial resolution imaging survey may reveal a faint WNE population, in brighter regions, shifting the distribution and making it more consistent with the Type Ib SN distribution in the brightest regions.

The KS test of the WC distribution, like the WN distribution, is most representative of the Type Ib distribution, which is consistent with a visual inspection. Again, the distribution deviates in the brightest 20 per cent of the galaxy, suggesting that incompleteness also plays a, albeit smaller, role for the WC population. Classically, we would expect the WC distribution to be most consistent with the Type Ic distributions, whereas the result presented here indicates that WC stars are possible progenitors of Type $\mathrm{Ib} \mathrm{SNe}$ as suggested by Georgy et al. (2009).

Note that both the WN and WC distributions do not favour a single ccSNe distribution across the entire fractional flux range. It is plausible that the WN-Type Ib and WC-Type Ic connections deviate from a one-to-one correlation, such that some WN and WC stars may produce Type II and Type Ib SNe, respectively.

However, we admit that the results presented in this section are based on small number statistics and to achieve a more significant result we require spectroscopic confirmation of the remaining WR candidates listed in Table 2, together with other on-going WR galaxy surveys.

\section{SUMMARY}

We present results from our VLT/FORS1 imaging and Gemini/GMOS spectroscopic survey of the WR population in the nearby star-forming galaxy NGC 5068. 
From our VLT/FORS1 narrow-band $\lambda 4684$ imaging we have identified 160 emission-line regions, for which we have obtained photometry. We obtained follow-up Gemini/GMOS spectroscopy of 67 of our photometric candidates, of which only 22 exhibited statistically significant $\lambda 4684$ excesses, while five were not detected in the $\lambda 4781$ continuum image. WR emission lines were identified in 30 candidates, of which 21 were statistically significant. Based on line luminosity calibrations based on LMC WR stars we spectroscopically confirmed $18 \mathrm{WN}$ and $24 \mathrm{WC}$ stars.

From comparison of the photometric properties of our confirmed WR stars with the remaining candidates we infer the presence of at least an additional $11 \mathrm{WC}$ stars, $43 \mathrm{WN}$ stars and approximately 12 WR stars of unknown subtype, bringing the total to $\sim 120 \mathrm{WR}$ stars. A comparison with the LMC WR stars degraded to a spatial resolution of $\sim 20 \mathrm{pc}$ suggests that our imaging is fairly complete, although faint WR sources with intrinsically small $m_{4684}-m_{4784}$ excesses, from which we estimate a global WR population of $\sim 170$ in NGC 5068

Our spectroscopy enables us to re-derive the metallicity gradient of NGC 5068 using a combination of strong line methods. We find

$\log (\mathrm{O} / \mathrm{H})+12=8.74 \pm 0.15-(0.61 \pm 0.22) r / R_{25}$,

revealing a strong metallicity gradient, contrary to the positive gradient found by Pilyugin et al. (2004).

Nebular spectroscopy revealed $\left[\mathrm{N}_{\mathrm{II}}\right] / \mathrm{H} \alpha=0.155$ which we applied to the published $\mathrm{H} \alpha$ flux of Kennicutt et al. (2008) to reveal a SFR $\sim 0.63_{-0.13}^{+0.11} \mathrm{M}_{\odot} \mathrm{yr}^{-1}$, revealing an $\mathrm{O}$ star population of $\sim 9000_{-2000}^{+1500}$, and $N(\mathrm{O}) / N(\mathrm{WR}) \sim 0.019_{-0.003}^{+0.005}$ based on $N(\mathrm{O}) / N(\mathrm{O} 7 \mathrm{~V}) \sim 1.5$. A comparison between our census of NGC 5068 and the survey of NGC 7793 by Bibby \& Crowther (2010) reveals that evolutionary predictions may overestimate the $N(\mathrm{WR}) / N(\mathrm{O})$ ratio for both galaxies by 50 per cent, unless we have significantly underestimated the fraction of faint, weak excess WR stars.

Finally, we assess the location of our spectroscopically confirmed WN and WC stars within NGC 5068 relative to the galaxy light and compare the distributions with those of different subtypes of ccSNe. We find that both WN and WC stars are most consistent with the Type Ib ccSNe distribution, however, both distributions, particularly WN stars, are more consistent with the Type II distribution in the brightest regions. However, for the WN stars this could be explained by the missing weaker WNE stars which would remain undetected in brighter regions.

\section{ACKNOWLEDGMENTS}

The authors thank the anonymous referee for careful reading of the manuscript and for suggestions which helped to improve the quality of the paper. JLB acknowledges financial support from STFC and also ongoing support from Hilary Lipsitz. Data presented in this paper are based on observations made with ESO Telescopes at the Paranal Observatories under programme ID 081.B-0289 and observations obtained at the Gemini Observatory, under program ID GS2009A-Q-20, which is operated by the Association of Universities for Research in Astronomy, Inc., under a cooperative agreement with the NSF on behalf of the Gemini partnership: the National Science Foundation (United States), the Science and Technology Facilities Council (United Kingdom), the National Research Council (Canada), CONICYT (Chile), the Australian Research Council (Australia), Ministério da Ciência e Tecnologia (Brazil) and Ministerio de Ciencia, Tecnología e Innovación Productiva (Argentina).

\section{REFERENCES}

Anderson J. P., James P. A., 2008, MNRAS, 390, 1527

Bibby J. L., Crowther P. A., 2010, MNRAS, 405, 2737

Cedres B., Urbaneja M. A., Cepa J., 2004, A\&A, 422, 511

Conti P. S., Massey P., 1989, ApJ, 337, 251

Conti P. S., Crowther P. A., Leitherer C., 2008, From Luminous Hot Stars to Starburst Galaxies. Cambridge Univ. Press, Cambridge

Crockett R. M. et al., 2007, MNRAS, 381, 835

Crowther P. A., 2007, ARA\&A, 45, 177

Crowther P. A., Hadfield L. J., 2006, A\&A, 449, 71

Crowther P. A., De Marco O., Barlow M. J., 1998, MNRAS, 296, 367

de Vaucouleurs G., de Vaucouleurs A., Corwin H. G., Jr, Buta R. J., Paturel

G., Fouque P., 1991, Third Reference Catalogue of Bright Galaxies. Springer-Verlag, Berlin

Eldridge J. J., Vink J. S., 2006, A\&A, 452, 295

Fruchter A. S. et al., 2006, Nat, 441, 463

Georgy C., Meynet G., Walder R., Folini D., Maeder A., 2009, A\&A, 502, 611

Hadfield L. J., Crowther P. A., 2007, MNRAS, 381, 418

Hadfield L. J., Crowther P. A., Schild H., Schmutz W., 2005, A\&A, 439, 265

Hamuy M., Suntzeff N. B., Heathcote S. R., Walker A. R., Gigoux P., Phillips M. M., 1994, PASP, 106, 566

Herrmann K. A., Ciardullo R., Feldmeier J. J., Vinciguerra M., 2008, ApJ, 683,630

Hodge P. W., 1974, PASP, 86, 845

Hummer D. G., Storey P. J., 1987, MNRAS, 224, 801

Karachentsev I. D., 2003, A\&A, 404, 93

Karachentsev I. D. et al., 2007, ApJ, 133, 504

Kelly P. L., Kirshner R. P., Pahre M., 2008, ApJ, 687, 1201

Kennicutt R. C., Jr, 1984, ApJ, 287, 116

Kennicutt R. C., Jr, 1998, ARA\&A, 36, 189

Kennicutt R. C., Jr, Lee J. C., Funes Jose G. S. J., Sakai S., Akiyama S., 2008, ApJS, 178, 247

Kewley L. J., Dopita M. A., 2002, ApJS, 142, 35

Kewley L. J., Ellison S. L., 2008, ApJ, 681, 1183

Langer N., Maeder A., 1995, A\&A, 295, 685

Leloudas G., Sollerman J., Levan A. J., Fynbo J. P. U., Malesani D., Maund J. R., 2010, A\&A, 518, 29

Li W., Chornock R., Leaman J., Filippenko A. V., Pozanski D., Wang X., Ganeshalingam M., Mannucci F., 2011, MNRAS, 412, 1473

McCall M. L., Rybski P. M., Shields G. A., 1985, ApJS, 57, 1

Maeder A., Meynet G., 2000, ARA\&A, 38, 143

Martin P., Friedli D., 1997, A\&A, 326, 449

Massey P., 1996, in Verux J. M., Detal A., Fraipont-Caro D., Gosset E., Rauw G., eds, Liege Int. Astrophys. Colloq. Vol. 33, Wolf-Rayet Stars in the Framework of Stellar Evolution. Univ. Liege, Liege, p. 361

Mazzali P. A. et al., 2002, ApJ, 572, L61

Meynet G., Maeder A., 2005, A\&A, 429, 581

Modjaz M., Kewley L., Bloom J. S., Filippenko A. V., Perley D., Silverman J. M., 2011, ApJ, 731, 4

Moffat A. J., Shara M. M., 1983, ApJ, 273, 544

Neugent K. F., Massey P., 2011, ApJ, 733, 123

Osterbrock D. E., 1989, Astrophysics of Gaseous Nebulae and Active Galactic Nuclei. University Science Books, Mill Valley, CA

Pagel B. E. J., Edmunds M. G., 1981, ARA\&A, 19, 77

Pettini M., Pagel B. E. K., 2004, MNRAS, 348, L59

Pilyugin L. S., Vilchez J. M., Contini T., 2004, A\&A, 425, 849

Podsialowski Ph., Joss P. C., Hsu J. J. L., 1992, ApJ, 391, 246

Rosa M., D’Odorica S., 1986, in De Loore C. W. H., Willis A. J., Laskandes P., eds, Proc. IAU Symp. 116, Luminous Stars and Associations in Galaxies. Reidel, Dordrecht, p. 355

Ryder S. D., 1995, ApJ, 444, 610

Ryder S. D., Dopita M. A., 1994, ApJ, 430, 142

Schaerer D., Vacca W. D., 1998, ApJ, 497, 618

Schild H., Crowther P. A., Abbott J. B., Schmutz W., 2003, A\&A, 397, 859 
Seaton M. J., 1979, MNRAS, 187, 73 ${ }^{\mathrm{pM}}$

Smartt S. J., 2009, ARA\&A, 47, 63

Smartt S. J., Maund J. R., Hendry M. A., Tout C. A., Gilmore G. F., Mattila S., Benn C. R., 2004, Sci, 303, 499

Smartt S. J., Eldridge J. J., Crockett R. M., Maund J. R., 2009, MNRAS, 395, 1409

Smith L. F., Shara M. M., Moffat A. F. J., 1996, MNRAS, 281, 163

Smith N., Li W., Filippenko A. V., Chornock R., 2011, MNRAS, 421, 1522

Stetson P. B., 2000, PASP, 112, 925

Tody D., 1986, Proc. SPIE, 627, 733

Vacca W. D., 1994, ApJ, 421, 140

Woosely S. E., Bloom J. S., 2006, ARA\&A, 44, 507

Zaritsky D., Kennicutt R. C., Jr, Huchra J. P., 1994, ApJ, 420, 87

\section{SUPPORTING INFORMATION}

Additional Supporting Information may be found in the online version of this article:

Appendix A. Finding charts.

Please note: Wiley-Blackwell are not responsible for the content or functionality of any supporting materials supplied by the authors. Any queries (other than missing material) should be directed to the corresponding author for the article.

This paper has been typeset from a $\mathrm{T}_{\mathrm{E}} \mathrm{X} / \mathrm{L} \mathrm{T} \mathrm{E} \mathrm{X}$ file prepared by the author. 